\title{
Gamow-Teller strength functions of superfluid odd nuclei and neutrino capture reactions
}

\author{
I.N. Borzov ${ }^{1 \dagger}$, S.A. Fayans ${ }^{2 \S}$ and E.L. Trykov ${ }^{1}$ \\ 1) Institute of Physics and Power Engineering, 249020 Obninsk, Russia \\ $\left.{ }^{2}\right)$ GANIL, B.P. 5027, F-14021 Caen Cedex, France
}

\begin{abstract}
:
The charge-exchange excitations of superfluid odd-A nuclei are studied within the framework of the self-consistent finite Fermi-system theory. The pairing blocking effect due to the odd quasiparticle, the effective $N N$-interactions both in the particlehole $(p h)$ and particle-particle $(p p)$ channel, and also the $p h$-continuum are taken into account. Gamow-Teller strength functions of ${ }^{71} \mathrm{Ga},{ }^{115} \mathrm{In}$ and ${ }^{19} \mathrm{~F}$ nuclei are calculated and compared with those extracted from experimental spectra of $(p, n)$ reactions. The total capture cross sections of the reactor electron antineutrinos are calculated (assuming complete $\bar{\nu}_{e} \rightarrow \nu_{e}$ conversion), as well as the solar neutrino capture rates for the ${ }^{71} \mathrm{Ga}$ and ${ }^{115} \mathrm{In}+{ }^{19} \mathrm{~F}$ detectors. Uncertainties regarding nuclear structure information involved in these calculations are discussed.
\end{abstract}

PACS numbers: 21.60, 25.30.Pt

Keywords: Structure of odd nuclei, solar neutrino capture rates.

† Present address: Max-Plank Institut für Astrophysik, 85740 Garching, FRG.

$\S$ On leave from Russian Research Centre - Kurchatov Institute, 123182 Moscow, Russia. 



\section{Introduction}

The study of charge-exchange modes of excitations of superfluid odd-A nuclei is not only important for better understanding of nuclear $\beta$-decay process but also has significant practical applications. In particular, the effect of the renormalization of the weak axial-vector current in nuclear media has drawn much attention. Such a renormalization leads to the observed quenching [1] of matrix elements for the lowenergy $\left(\omega<\varepsilon_{F}\right)$ spin-isospin-flip transitions in nuclei. The microscopic analyses of the $(p, n)$ and $(n, p)$ reactions at intermediate energies have showed that only $50-65 \%$ of the Ikeda model-independent sume rule $3(N-Z)$ for the Gamow-Teller (GT) strength can be extracted from experimental spectra for $A>40$ nuclei $[2,3,4]$. Shell-model calculations of the $\beta$-decay GT matrix elements in $s d$-nuclei yielded an estimated overall quenching factor of $0.56(5)$ [5]. Additional information about the quenching effect has been obtained from experiments on high-energy $\beta^{+}$-decay of some neutron-deficient nuclei [6]. The GT strength functions for those nuclei can be measured in a wider energy range due to the higher $Q_{\beta}$-values. The $\beta$ decay data substantially complement those extracted from charge-exchange reactions. The striking examples are recent measurements of allowed $\beta$-transitions in some new $f p$-nuclides: ${ }^{48} \mathrm{Mn}$ with $Q_{\beta}=13.6 \mathrm{MeV}[7]$ and ${ }^{37} \mathrm{Ca}$ with $Q_{\beta}=11.64 \mathrm{MeV}$ [8]. Evidence for the possible $A$-dependence of the quenching factor has been found in the systematics of matrix elements of mirror ground state transitions in odd nuclei [9].

For the theoretical analyses of GT strength functions in odd nuclei multi-configurational shell models are widely used $[10,11]$. An advantage of these models is that complex configurations can be included more efficiently than in the QRPA-like scheme [12]. However, such shell-model approaches suffer from the sensitivity to the basis truncation which is technically unavoidable for medium and heavy nuclei. Therefore the conclusions about the absence of $g_{A} / g_{V}$ renormalization in nuclear medium drawn from recent shell-model analysis of the high-energy $\beta$-decay of ${ }^{37} \mathrm{Ca}[8]$ have to be taken with some care (see Refs. $[13,14]$ ).

The practical aspect of the problem concerns solar neutrino [15] and reactorantineutrino capture rates in detectors based on the inverse $\beta^{ \pm}$-decay processes, i.e. on the $\nu(\bar{\nu})$-capture reactions, which take place due to the electroweak charge currents,

$$
\begin{aligned}
& \nu_{e}+A_{Z}\left(I^{\pi}\right) \rightarrow e^{-}+A_{Z+1}\left(I^{\prime \pi^{\prime}}\right), \\
& \bar{\nu}_{e}+A_{Z}\left(I^{\pi}\right) \rightarrow e^{+}+A_{Z-1}\left(I^{\prime \pi^{\prime}}\right) .
\end{aligned}
$$

A number of nuclei were suggested for neutrino detectors. The most promising ones are the odd-A nuclei with very low reaction thresholds: ${ }^{71} \mathrm{Ga}\left(Q_{\beta}=-236 \mathrm{keV}\right)$, ${ }^{81} \mathrm{Br}(-471 \mathrm{keV}),{ }^{115} \mathrm{In}(-120 \mathrm{keV})$ which are sensitive both to low and to high energy portions of the solar neutrino spectrum.

Recently, the interest in the reactions (1) and (2) has increased because of on-going SAGE [16] and GALLEX [17] experiments and of proposal of the ${ }^{115} \mathrm{In}+{ }^{19} \mathrm{~F}$ detector [18]. A distinct feature of the latter could be a higher detection efficiency for solar pp 
neutrinos of ${ }^{115} \mathrm{In}$ and a sensitivity of ${ }^{19} \mathrm{~F}$ to the boron neutrinos detected. The matrix element of the ${ }^{19} \mathrm{~F}\left(\frac{1}{2}^{+}\right) \rightarrow{ }^{19} \mathrm{Ne}\left(\frac{1}{2}^{+}\right)$transition between analog states is known experimentally. The unique signature of $\gamma$ transitions in the daughter nucleus, ${ }^{115} \mathrm{Sn}$, could provide a low-background regime of a direct counting experiment, giving a promising possibility of studying the solar neutrino. An observation of the ${ }^{19} \mathrm{~F} \rightarrow{ }^{19} \mathrm{O}$ reaction might support the idea of resonant spin-flavor neutrino conversion inside the Sun [19]. The In-F detector can be also used to searching for the anomalous reactor antineutrino events, namely ${ }^{115} \operatorname{In}\left(\bar{\nu}_{e}, e^{-}\right){ }^{115} \mathrm{Sn}$ and ${ }^{19} \mathrm{~F}\left(\bar{\nu}_{e}, e^{-}\right){ }^{19} \mathrm{Ne}$, which could provide information about $\bar{\nu}_{e} \leftrightarrow \nu_{e}$ oscillations. The ratio of the experimental upper limits on these reactions to the cross sections calculated in the $\bar{\nu}_{e} \equiv \nu_{e}$ approximation (full $\bar{\nu}_{e} \rightarrow \nu_{e}$ conversion) would give constraints on the mixing of $\bar{\nu}$ and $\nu$ states.

The reliable estimate of the cross sections for solar neutrino and reactor antineutrino capture reactions requires the microscopic calculation of the $\beta$-decay strength functions, with the Fermi and Gamow-Teller ones being most important. A number of recent works has been devoted to such calculations. In Ref. [20], the multiconfigurational shell model was used with an effective $G$-matrix interaction. The strength functions were calculated using "zero approximation of the shell model" in a complex configuration space (from $2 p 1 h$ to $5 p 1 h$ ). In Ref. [21], the HF+BCS approach was employed with a phenomenological Yukawa-type interaction and adjustable parameters within an extended configurational space (one- and three-quasiparticle states were taken into account). In Ref. [22], the theory of finite Fermi systems (FFS) [23] with zero-range effective interaction in a truncated $p h$-space and schematic pairing model was used. In Ref. [24], an optical shell-model version of the FFS theory including pairing correlations, effective $p h$-interaction and $p h$-continuum was employed. In the present paper we shall apply a more elaborated approach to the charge-exchange excitations in superfluid odd nuclei, based on the self-consistent density-functional FFS theory which, in particular, incorporates the blocking effect, the $p h$-continuum and effective interactions both in the $p h$ - and $p p$-channels.

The plan of the paper is as follows. In Sect. 2, we describe the formalism of our approach. In Sect. 3, the results of the GT strength function calculations and capture rates of reactor antineutrinos and solar neutrinos on ${ }^{71} \mathrm{Ga},{ }^{115} \mathrm{In}$ and ${ }^{19} \mathrm{~F}$ are presented and discussed. Sect. 4 contains our main conclusions.

\section{Self-consistent description of charge-exchange excitations in superfluid odd nuclei}

The system of the FFS theory equations [23] for collective charge-exchange excitations in non-magic nuclei can be represented as

$$
\begin{array}{ll}
V_{p n}=e_{q} V_{p n}^{0}+\sum_{n^{\prime} p^{\prime}} F_{n p, n^{\prime} p^{\prime}}^{\omega} \rho_{p^{\prime} n^{\prime}}, & d_{p n}^{1}=\sum_{n^{\prime} p^{\prime}} F_{n p, n^{\prime} p^{\prime}}^{\xi} \varphi_{p^{\prime} n^{\prime}}^{1}, \\
V_{p n}^{h}=\sum_{n^{\prime} p^{\prime}}^{h} F_{n p, n^{\prime} p^{\prime}}^{\omega} \rho_{p^{\prime} n^{\prime}}^{h}, & d_{p n}^{2}=\sum_{n^{\prime} p^{\prime}} F_{n p, n^{\prime} p^{\prime}}^{\xi} \varphi_{p^{\prime} n^{\prime}}^{2}
\end{array}
$$


Here $V_{p n}$ and $V_{p n}^{h}$ are the effective fields for particles and holes, respectively, arising under an action of the external charge-exchange field $V_{0} \propto \tau_{ \pm} ; d^{1}$ and $d^{2}$ are the effective changes of the corresponding pairing fields; $F^{\omega}$ and $F^{\xi}$ are the effective interactions in the $p h$ - and $p p$-channels; $\rho, \rho^{h}, \varphi^{1}$ and $\varphi^{2}$ are the corresponding transition densities for the charge-exchange excitations $\left(V_{p n}^{0 h}=0\right)$. In (3), an expansion over single-particle wave functions $\varphi_{\lambda}{ }^{\top}$ is implied: the so called $\lambda$-representation with $\lambda^{n(p)}=n l s j m \tau$ being the standard set of single-particle quantum numbers of the neutron $(\tau=\nu)$ or proton $(\tau=\pi)$ level with energy $\left.\varepsilon_{\lambda}^{\tau}\right]$. Because of the continuum it is practically impossible to solve these equations in the $\lambda$-representation without basis truncation. To overcome this difficulty, a method of including the whole $p h$-continuum in the FFS theory equations for the charge-exchange excitations of superfluid nuclei was developed $[12,25]$ which is similar to the method of mixed $(r, \lambda)$-representation used in Ref. [26] for the neutral excitations.

The essential feature of the approach utilized in the present paper is a selfconsistent description of the ground and excited states of even and odd nuclei with pairing correlations. Accordingly, the calculational scheme consists of two steps:

1) constructing the self-consistent potential and the quasiparticle basis;

2) solving the QRPA-type equations and calculating the strength functions of nuclear charge-exchange excitations.

For a self-consistent description of the excited states in non-magic nuclei one needs first to obtain the mean fields for neutrons and protons with properly taking into account the effects of pairing correlations. We employ the density functional method and the quasiparticle Hamiltonian with a free kinetic energy operator, so that the quasiparticle effective mass $m^{*}$ equals to the bare nucleon mass $m\left(m / m^{*}=1\right)$. In this approach, the nucleon density, $\rho$, is written as a sum of single-particle contributions, i.e., as a sum which minimizes the energy of the system. The quasiparticle spectrum and wave functions are calculated in a self-consistent mean field which is the first functional derivative of the density functional. Since the effective mass of nucleons on the Fermi surface in atomic nuclei is empirically known to be close to the bare mass, one expects that the calculated spectrum of the quasiparticle levels will be close to the observed one. Insofar, one may hope to succeed in describing the low-lying particle-hole collective states. These states are treated within the quasiparticle RPA approach, with the effective interactions $F^{\omega}$ and $F^{\xi}$ being the second derivatives of the same functional with respect to the normal and abnormal density, respectively. In fact, this method is a version of the self-consistent FFS theory, that has much in common with the HFB method with effective forces. The main problem of its practical application is the appropriate choice of the form and parametrization of the density functional. Here we shall use the density functional in the form suggested in Ref. [27], where the dependence on $\rho$ is simulated by simple fractional-linear functions and the surface contribution is related to the finite-range forces. Thus the interaction energy density is represented as

$$
\varepsilon_{\mathrm{int}}=\varepsilon_{\mathrm{main}}+\varepsilon_{\mathrm{coul}}+\varepsilon_{s l}+\varepsilon_{\mathrm{pair}}
$$


where

$$
\varepsilon_{\text {main }}=\frac{2}{3} \epsilon_{F}^{0} \rho_{0}\left[a_{+}^{v} x_{+}^{2} f_{+}^{v}+a_{-}^{v} x_{-}^{2} f_{-}^{v}+a_{+}^{s} x_{+} f_{+}^{s} \widetilde{f_{+}^{s} x_{+}}+a_{-}^{s} x_{-} f_{-}^{s} \widetilde{f_{-}^{s} x_{-}}\right] .
$$

Here, $x_{ \pm}=\left(\rho_{n} \pm \rho_{p}\right) / 2 \rho_{0}, \rho_{n(p)}$ is the neutron (proton) density, $2 \rho_{0}$ is the equilibrium nuclear matter density $(N=Z), \epsilon_{F}^{0}$ is the nuclear matter Fermi energy, and

$$
\begin{gathered}
f_{ \pm}^{v}=\frac{1-h_{1 \pm}^{v} x_{+}}{1+h_{2 \pm}^{v} x_{+}}, f_{ \pm}^{s}=\frac{1}{1+h_{ \pm}^{s} x_{+}}, \\
\widetilde{f_{ \pm}^{s} x_{ \pm}}=\int D\left(\vec{r}-\vec{r}^{\prime}\right) f_{ \pm}^{s}\left(\vec{r}^{\prime}\right) x_{ \pm}\left(\vec{r}^{\prime}\right) d \vec{r}^{\prime}
\end{gathered}
$$

where

$$
D\left(\vec{r}-\vec{r}^{\prime}\right)=\delta\left(\vec{r}-\vec{r}^{\prime}\right)-\frac{1}{4 \pi R^{2}\left|\vec{r}-\vec{r}^{\prime}\right|} \exp \left(\frac{-\left|\vec{r}-\vec{r}^{\prime}\right|}{R}\right) .
$$

In the momentum representation,

$$
D(q)=-\frac{(q R)^{2}}{1+(q R)^{2}},
$$

so that the two last terms in Eq. (5) correspond to the contributions of the surface isoscalar and isovector potential energies generated by the density-dependent finiterange forces. The energy density of the Coulomb interaction $\varepsilon_{\text {coul }}$ has the usual form including the exchange part in the Slater approximation,

$$
\varepsilon_{\text {coul }}=2 \pi e^{2} \rho_{p}(r)\left(\frac{1}{r} \int_{0}^{r} \rho_{p}(r) r^{2} d r+\int_{r}^{\infty} \rho_{p}(r) r d r\right)-\frac{3}{4}\left(\frac{3}{\pi}\right)^{1 / 3} e^{2} \rho_{p}^{4 / 3}(r) .
$$

The spin-orbit term $\varepsilon_{s l}$ in Eq. (4) comes from spin-orbit $\propto\left(\kappa+\kappa^{\prime} \vec{\tau}_{1} \cdot \vec{\tau}_{2}\right)\left[\vec{\nabla}_{1} \delta\left(\vec{r}_{1}-\right.\right.$ $\left.\left.\vec{r}_{2}\right) \times\left(\vec{p}_{1}-\vec{p}_{2}\right)\right] \cdot\left(\vec{\sigma}_{1}+\vec{\sigma}_{2}\right)$ and velocity spin-dependent $\propto\left(g_{1}+g_{1}^{\prime} \vec{\tau}_{1} \cdot \vec{\tau}_{2}\right)\left(\vec{\sigma}_{1} \cdot \vec{\sigma}_{2}\right)\left(\vec{p}_{1} \cdot \vec{p}_{2}\right)$ interactions. For spherical nuclei this term can be expressed through the spin-orbit densities,

$$
\rho_{s l}^{n, p}(\vec{r})=\sum_{\lambda} n_{\lambda}<\vec{\sigma} \cdot \vec{l}>_{\lambda}\left|\varphi_{\lambda}^{n, p}(\vec{r})\right|^{2}
$$

where $n_{\lambda}$ is an occupation number of the single-particle level $\lambda, \varphi_{\lambda}$ is its nucleon wave function and $\langle\vec{\sigma} \cdot \vec{l}\rangle_{\lambda}=j(j+1)-l(l+1)-3 / 4$. For the corresponding energy density one obtains

$$
\varepsilon_{s l}=C_{0} r_{0}^{2} \sum_{i, k=n, p}\left(\frac{1}{r} \rho_{s l}^{i} \kappa^{i k} \frac{d \rho}{d r}+\frac{1}{4 r^{2}} \rho_{s l}^{i} g_{1}^{i k} \rho_{s l}^{k}\right),
$$

where $\kappa^{n n}=\kappa^{p p}=\kappa+\kappa^{\prime}, \kappa^{n p}=\kappa^{p n}=\kappa-\kappa^{\prime} ; g_{1}^{n n}=g_{1}^{p p}=g_{1}+g_{1}^{\prime}, g_{1}^{n p}=g_{1}^{p n}=g_{1}-g_{1}^{\prime}$; $C_{0}=2 \varepsilon_{F}^{0} / 3 \rho_{0}, r_{0}=\left(3 / 8 \pi \rho_{0}\right)^{1 / 3}$. The last term in Eq. (4), the pairing energy density $\varepsilon_{\text {pair }}$, is represented as

$$
\varepsilon_{\mathrm{pair}}=\frac{1}{2} \nu F^{\xi} \nu
$$


where $\nu$ is the anomalous nucleon density and $F^{\xi}$ plays the role of effective force in the particle-particle channel. This force, both for neutrons and protons, in the present paper is chosen in the simplest form,

$$
F^{\xi}=-C_{0} f^{\xi} \delta\left(\vec{r}-\vec{r}^{\prime}\right) .
$$

Here, $f^{\xi}$ is a dimensionless interaction constant of the FFS theory [23]. The superscript $\xi$ refers to the energy cut-off parameter which defines the number of singleparticle levels taken into account when evaluating the anomalous Green's functions and, correspondingly, when solving the equations for pairing fields $\Delta(\vec{r})$ and chemical potentials $\mu$ as well as the dynamical FFS equations for excited states.

Thus the total interaction energy of the superfluid nucleus, $E_{\text {int }}[\rho, \nu]=\int \mathrm{d} \vec{r} \varepsilon_{\text {int }}(\vec{r})$, where $\varepsilon_{\text {int }}(\vec{r})$ is defined as above, is a functional of two densities, the normal, $\rho(\vec{r})$ , and the anomalous, $\nu(\vec{r})$. Self-consistent calculation with such a functional looks like the standard variational HFB procedure in which the single-particle Hamiltonian takes the form

$$
\mathcal{H}=\left(\begin{array}{cc}
h-\mu & -\Delta \\
-\Delta & \mu-h
\end{array}\right)
$$

where

$$
h=\frac{p^{2}}{2 m}+\frac{\delta E[\rho, \nu]}{\delta \rho}, \Delta=-\frac{\delta E[\rho, \nu]}{\delta \nu} .
$$

These equations have been solved iteratively as follows. For given densities $\left(\rho^{(i)}, \nu^{(i)}\right)$, from the above functional, Eq. (4), the elements of the Hamiltonian $\mathcal{H}^{(i)}$ were derived, through its eigenvalues and wave functions $\left(u^{(i)}, v^{(i)}\right)$ the new densities were calculated, and then, as an input $\left(\rho^{(i+1)}, \nu^{(i+1)}\right)$ for the next iteration, superpositions of previous densities and these new ones were used with the weights of 0.85 and 0.15 , respectively. This procedure continued until complete convergence was achieved. $\mathrm{Pa}$ rameters of the density functional, Eq. (4), were chosen by fitting binding energies, charge distributions and single-particle spectra for magic nuclei ${ }^{40} \mathrm{Ca},{ }^{48} \mathrm{Ca},{ }^{208} \mathrm{~Pb}$ and for non-magic ones both with weak superfluidity $\left({ }^{90} \mathrm{Zr},{ }^{146} \mathrm{Gd}\right)$ and developed pairing (even-even $\mathrm{Sn}$ and $\mathrm{Pb}$ isotopes). Pairing was treated in diagonal approximation within the basis of all bound single-particle levels. To reduce the number of adjustable parameters in the fitting procedure, it was assumed that $f_{-}^{v}=f_{+}^{v}$ (i.e. $h_{1-}^{v}=h_{1+}^{v}$ and $\left.h_{2-}^{v}=h_{2+}^{v}\right)$, and the surface symmetry energy was neglected $\left(a_{-}^{s}=0\right)$. As a result the following set of parameters was deduced

$$
\left.\begin{array}{c}
a_{+}^{v}=-7.391, h_{1+}^{v}=0.037, h_{2+}^{v}=1.322, \\
a_{-}^{v}=3.595 \\
a_{+}^{s}=10.0, h_{2+}^{s}=0.31 \\
\kappa^{p p}=\kappa^{p n}=0.205 \\
g_{1}^{p p}=-g_{1}^{p n}=-0.11 \\
f^{\xi}=0.33 \\
R=0.35 \mathrm{fm}, r_{0}=1.135 \mathrm{fm}
\end{array}\right\}
$$


We notice the following relations between a number of parameters and the nuclear matter characteristics:

$$
\left.\begin{array}{c}
a_{+}^{v}=\alpha+(5 \alpha+6) / 5 \eta \\
h_{1+}^{v}=1-\alpha / a_{+}^{v} \eta \\
h_{2+}^{v}=1 / \eta-1 \\
=\left(3 \beta^{0} / \epsilon_{F}^{0}-1\right) / f_{-}^{v}\left(x_{+}=1\right)
\end{array}\right\}
$$

where

$$
\alpha=3 \frac{\mu^{0}}{\epsilon_{F}^{0}}-\frac{9}{5}, \quad \eta=\frac{5 K^{0}+6 \epsilon_{F}^{0}}{18\left(\epsilon_{F}^{0}-5 \mu^{0}\right)} .
$$

Here, the infinite nuclear matter parameters have the following meaning: $\mu^{0}$ is the chemical potential (binding energy per one nucleon), $K^{0}$ the compression modulus, $\epsilon_{F}^{0}$ the Fermi energy, and $\beta^{0}$ the symmetry energy. The deduced functional parameters correspond to $\mu^{0}=-15.73 \mathrm{MeV}, K^{0}=135 \mathrm{MeV}, \epsilon_{F}^{0}=37.35 \mathrm{MeV}$ and $\beta^{0}=31$ $\mathrm{MeV}$. In what follows, the mean-field potential constructed in this way is used for a description of different excited states.

In our calculations the pairing potential $\Delta$ is a smooth function of $\vec{r}$ and its nondiagonal matrix elements $\Delta_{\lambda \lambda^{\prime}}$ are small, i.e. $\Delta_{\lambda \lambda^{\prime}} \approx \Delta_{\lambda} \delta_{\lambda \lambda^{\prime}}$. The level-dependent matrix elements $\Delta_{\lambda}$ can be obtained from the standard equation

$$
\Delta_{\lambda}^{\tau}=\sum_{\lambda^{\prime}} F_{\lambda \lambda, \lambda^{\prime} \lambda^{\prime}}^{\xi} \frac{\Delta_{\lambda^{\prime}}^{\tau}\left(2 j_{\lambda^{\prime}}+1\right)}{2 E_{\lambda^{\prime}}^{\tau}},
$$

which is to be solved toghether with the condition on the chemical potential $\mu^{\tau}$

$$
N_{\tau}=\sum_{\lambda} \frac{E_{\lambda}-\left(\varepsilon_{\lambda}-\mu\right)}{2 E_{\lambda}}\left(2 j_{\lambda}+1\right),
$$

where $N_{\nu}$ and $N_{\pi}$ are the neutron and proton number, respectively, and

$$
E_{\lambda}=\sqrt{\left(\varepsilon_{\lambda}-\mu\right)^{2}+\Delta_{\lambda}^{2}}
$$

Here, the $\lambda$ set does not include magnetic quantum number $m$ and the index $\tau$ is omitted for simplicity in the r.h.s. of Eq. (21). The fact that the pairing affects only a small number of levels in the vicinity of the Fermi surface allows the mixed $(r, \lambda)$-representation technique to be developed.

The situation in odd nuclei is somewhat different since in the states $\lambda_{0}\left(-\lambda_{0}\right)$ no additional quasiparticle (quasihole) can be created. The gap equation (20) is now written in the form:

$$
\Delta_{\lambda}^{\tau}=\sum_{\lambda^{\prime} \neq \lambda_{0}} F_{\lambda \lambda, \lambda^{\prime} \lambda^{\prime}}^{\xi} \frac{\Delta_{\lambda^{\prime}}^{\tau}\left(2 j_{\lambda^{\prime}}+1\right)}{2 E_{\lambda^{\prime}}^{\tau}}+F_{\lambda \lambda_{1} \lambda_{0} \lambda_{0}}^{\xi} \frac{\Delta_{\lambda_{0}}^{\tau}\left(2 j_{\lambda_{0}}-1\right)}{2 E_{\lambda_{0}}^{\tau}} .
$$

and for the particle number in the ground state one gets

$$
N_{\tau}=1+\left(2 j_{\lambda_{0}}-1\right) v_{\lambda_{0}}^{2}+\sum_{\lambda^{\prime} \neq \lambda_{0}} v_{\lambda^{\prime}}^{2}\left(2 j_{\lambda}^{\prime}+1\right),
$$


where the occupation factors are given by

$$
v_{\lambda}^{2}=\frac{E_{\lambda}-\left(\varepsilon_{\lambda}-\mu\right)}{2 E_{\lambda}}
$$

However, the odd quasiparticle does affect not only ground state properties. Let us write, besides the effective field equations (3), the conjugate equations for chargeexchange transition densities in the even nucleus [23]:

$$
\begin{aligned}
& \rho_{p n}=L_{p n}(\omega) V_{p n}+M_{p n}(\omega) V_{p n}^{h}+N_{p n}^{1}(\omega) d_{p n}^{1}+N_{p n}^{2}(\omega) d_{p n}^{2}, \\
& \rho_{p n}^{h}=M_{p n}(\omega) V_{p n}+L_{p n}(-\omega) V_{p n}^{h}+N_{p n}^{2}(-\omega) d_{p n}^{1}+N_{p n}^{1}(-\omega) d_{p n}^{2}, \\
& \varphi_{p n}^{1}=N_{p n}^{1}(\omega) V_{p n}+N_{p n}^{2}(-\omega) V_{p n}^{h}+K_{p n}(\omega) d_{p n}^{1}-M_{p n}(\omega) d_{p n}^{2}, \\
& \varphi_{p n}^{2}=N_{p n}^{2}(\omega) V_{p n}+N_{p n}^{1}(-\omega) V_{p n}^{h}-M_{p n}(\omega) d_{p n}^{1}+K_{p n}(-\omega) d_{p n}^{2} .
\end{aligned}
$$

Here $\omega=\tilde{\omega}-\delta \mu$, where $\delta \mu=\mu^{p}-\mu^{n}$ is the difference between the proton and the neutron chemical potentials, and $\tilde{\omega}$ is the nuclear excitation energy. Propagators obtained by integrating various products of the normal and abnormal Green's functions $G$ and $F$ in the $\lambda$-representation [23] are written as

$$
\begin{gathered}
L_{p n}(\omega)=\int G_{p}(\varepsilon) G_{n}(\varepsilon+\tilde{\omega}) \frac{d \varepsilon}{2 \pi i}=-\left[\frac{v_{p}^{2} u_{n}^{2}}{E_{p n}-\omega}+\frac{v_{n}^{2} u_{p}^{2}}{E_{p n}+\omega}\right], \\
M_{p n}(\omega)=-\int F_{p}(\varepsilon) F_{n}(\varepsilon+\tilde{\omega}) \frac{d \varepsilon}{2 \pi i}=-\frac{\Delta_{p} \Delta_{n}}{4 E_{p} E_{n}}\left[\frac{1}{E_{p n}-\omega}+\frac{1}{E_{p n}+\omega}\right], \\
N_{p n}^{1}(\omega)=-\int G_{p}(\varepsilon) F_{n}\left(\epsilon+\tilde{\omega} \frac{d \varepsilon}{2 \pi i}=-\frac{\Delta_{n}}{2 E_{n}}\left[\frac{v_{p}^{2}}{E_{p n}-\omega}-\frac{u_{p}^{2}}{E_{p n}+\omega}\right],\right. \\
N_{p n}^{2}(\omega)=-\int F_{p}(\varepsilon) G_{n}(\varepsilon+\bar{\omega}) \frac{d \varepsilon}{2 \pi i}=\frac{\Delta_{p}}{2 E_{p}}\left[\frac{u_{n}^{2}}{E_{p n}-\omega}-\frac{v_{n}^{2}}{E_{p n}+\omega}\right], \\
K_{p n}(\omega)=-\int G_{p}(\varepsilon) G_{n}^{h}(\varepsilon+\tilde{\omega}) \frac{d \epsilon}{2 \pi i}=-\left[\frac{v_{p}^{2} v_{n}^{2}}{E_{p n}-\omega}+\frac{u_{p}^{2} u_{n}^{2}}{E_{p n}+\omega}\right],
\end{gathered}
$$

where the notation $E_{p n}=E_{p}+E_{n}$ is used. The positive frequencies $\omega$ correspond to the states in the $\beta^{+}$-decay channel excited in the $(n, p)$-type reactions and in the $\bar{\nu}_{e}$-capture processes while the negative frequencies correspond to the states in the $\beta^{-}$-channel excited in the $(p, n)$-type reactions and in the $\nu_{e}$-capture processes.

Let the odd quasiparticle be in the proton (neutron) state $p_{0}\left(n_{0}\right)$. The singleparticle Green's functions for this state change, as well as the corresponding propagators, since now their poles belong to the upper energy half-plane:

$$
G_{p_{0}}(\varepsilon)=\frac{v_{p_{0}}^{2}}{\varepsilon-\mu_{p}+E_{p_{0}}-i \gamma}+\frac{u_{p_{0}}^{2}}{\varepsilon-\mu_{p}-E_{p_{0}}-i \gamma},
$$




$$
\begin{gathered}
F_{p_{0}}(\varepsilon)=\frac{\Delta_{p_{0}}}{2 E_{p_{0}}}\left[\frac{1}{\varepsilon-\mu_{p}+E_{p_{0}}-i \gamma}-\frac{1}{\varepsilon-\mu_{p}-E_{p_{0}}-i \gamma}\right], \\
G_{p_{0}}^{h}(\varepsilon)=-\left[\frac{v_{p_{0}}^{2}}{\varepsilon-\mu_{p}+E_{p_{0}}-i \gamma}+\frac{u_{p_{0}}^{2}}{\varepsilon-\mu_{p}-E_{p_{0}}-i \gamma}\right] .
\end{gathered}
$$

The propagators incorporating this odd particle become

$$
\begin{aligned}
& L_{p_{0} n}(\omega)=\int G_{p_{0}}(\varepsilon) G_{n}(\varepsilon+\bar{\omega}) \frac{d \varepsilon}{2 \pi i}=-\left[\frac{v_{p_{0}}^{2} u_{n}^{2}}{\left(E_{n}+E_{p_{0}}-\omega\right.}+\frac{u_{p_{0}}^{2} u_{n}^{2}}{\left(E_{n}-E_{p_{0}}\right)-\omega}\right], \\
& L_{p_{0} n}(-\omega)=\int G_{p_{0}}^{h}(\varepsilon) G_{n}^{h}(\varepsilon+\bar{\omega}) \frac{d \varepsilon}{2 \pi i}=-\left[\frac{u_{p_{0}}^{2} v_{n}^{2}}{\left(E_{n}+E_{p_{0}}\right)-\omega}+\frac{v_{p_{0}}^{2} v_{n}^{2}}{\left(E_{n}-E_{p_{0}}\right)-\omega}\right], \\
& M_{p_{0} n}(\omega)=-\int F_{p_{0}}(\varepsilon) F_{n}(\varepsilon+\bar{\omega}) \frac{d \varepsilon}{2 \pi i}=-\frac{\Delta_{p_{0}} \Delta_{n}}{4 E_{p_{0}} E_{n}}\left[\frac{1}{\left(E_{n}+E_{p_{0}}\right)-\omega}-\frac{1}{\left(E_{n}-E_{p_{0}}\right)-\omega}\right], \\
& N_{p_{0} n}^{1}(\omega)=-\int G_{p_{0}}(\varepsilon) F_{n}(\varepsilon+\tilde{\omega}) \frac{d \varepsilon}{2 \pi i}=-\frac{\Delta_{n}}{2 E_{n}}\left[\frac{v_{p_{0}}^{2}}{\left(E_{n}+E_{p_{0}}\right)-\omega}+\frac{u_{p_{0}}^{2}}{\left(E_{n}-E_{p_{0}}\right)-\omega}\right] \\
& N_{p_{0} n}^{2}(\omega)=-\int F_{p_{0}}(\varepsilon) G_{n}(\varepsilon+\tilde{\omega}) \frac{d \varepsilon}{2 \pi i}=\frac{\Delta_{p_{0}} u_{n}}{2 E_{p_{0}}}\left[\frac{1}{\left(E_{n}+E_{p_{0}}\right)-\omega}-\frac{1}{\left(E_{n}-E_{p_{0}}\right)-\omega}\right], \\
& N_{p_{0} n}^{2}(-\omega)=-\int F_{p_{0}}(\varepsilon) G_{n}^{h}(\varepsilon+\bar{\omega}) \frac{d \varepsilon}{2 \pi i}=-\frac{\Delta_{p_{0}} v_{n}}{2 E_{p_{0}}}\left[\frac{1}{\left(E_{n}+E_{p_{0}}\right)-\omega}-\frac{1}{\left(E_{n}-E_{p_{0}}\right)-\omega}\right] \\
& N_{p_{0} n}^{1}(-\omega)=-\int G_{p_{0}}^{h}(\varepsilon) F_{n}(\varepsilon+\tilde{\omega}) \frac{d \varepsilon}{2 \pi i}=\frac{\Delta_{n}}{2 E_{n}}\left[\frac{u_{p_{0}}^{2}}{\left(E_{n}+E_{p_{0}}\right)-\omega}+\frac{v_{p_{0}}^{2}}{\left(E_{n}-E_{p_{0}}\right)-\omega}\right] \\
& K_{p_{0} n}(\omega)=-\int G_{p_{0}}(\varepsilon) G_{n}^{h}(\varepsilon+\bar{\omega}) \frac{d \varepsilon}{2 \pi i}=-\left[\frac{v_{p_{0}}^{2} v_{n}^{2}}{\left(E_{n}+E_{p_{0}}\right)-\omega}+\frac{u_{p_{0}}^{2} v_{n}^{2}}{\left(E_{n}-E_{p_{0}}\right)-\omega}\right] \\
& K_{p_{0} n}(-\omega)=-\int G_{p_{0}}^{h}(\varepsilon) G_{n}(\varepsilon+\bar{\omega}) \frac{d \varepsilon}{2 \pi i}=-\left[\frac{u_{p_{0}}^{2} u_{n}^{2}}{\left(E_{n}+E_{p_{0}}\right)-\omega}+\frac{v_{p_{0}}^{2} u_{n}^{2}}{\left(E_{n}-E_{p_{0}}\right)-\omega}\right] .
\end{aligned}
$$

The difference between the pole terms of $A_{p_{0} n}$ and $A_{p n}$ leads to the additional transitions due to existence of odd particle. Note that Eq. (26) with propagators (27) and (29) describes the excited states averaged over all possible spins of daughter 
nuclei. A more detailed description requires an extension of the configuration space. As for the continuum, it can be seen that the contribution of states far from the Fermi-surface is essential only for the $L_{p n}$ and $L_{p_{0} n}$ propagators. They go over to the corresponding propagators for the system without pairing at $\left|\varepsilon_{\lambda}-\mu\right|,\left|\varepsilon_{\lambda^{\prime}}-\mu\right| \gg \Delta$ while the other ones, $M, N^{1}, N^{2}$ and $K$, give negligible contribution in this limit. The $L$ propagator is calculated in the $(r, \lambda)$-representation by using the following recipe $[12,25,26]$ :

$$
L\left(\vec{r}, \vec{r}^{\prime} ; \omega\right)=A\left(\vec{r}, \vec{r}^{\prime} ; \omega\right)+\sum\left[L_{p n}(\omega)-\tilde{A}_{p n}(\omega)\right] \varphi_{n}^{*}\left(\vec{r}_{1}\right) \varphi_{p}\left(\vec{r}_{1}\right) \varphi_{n}\left(\vec{r}_{2}\right) \varphi_{p}^{*}\left(\vec{r}_{2}\right),
$$

where the propagator $A\left(\vec{r}_{1} ; \vec{r}_{2} ; \omega\right)$ can be calculated exactly. It is written as

$$
\begin{aligned}
A\left(\vec{r}_{1}, \vec{r}_{2} ; \omega\right) & =\sum_{n} v_{n}^{2} G_{p}\left(\vec{r}_{1}, \vec{r}_{2} ; \mu_{n}-E_{n}-\omega\right) \varphi_{n}^{*}\left(\vec{r}_{1}\right) \varphi_{n}\left(\vec{r}_{2}\right) \\
& +\sum_{p} v_{p}^{2} G_{n}\left(\vec{r}_{1}, \vec{r}_{2} ; \mu_{p}-E_{p}+\omega\right) \varphi_{p}^{*}\left(\vec{r}_{1}\right) \varphi_{p}\left(\vec{r}_{2}\right) .
\end{aligned}
$$

Here the Green's functions $G_{p(n)}$ can be expressed in closed form in terms of regular and irregular solutions of the Schrödinger equation so that the ph-continuum is completely taken into account [28]. The "pairing" part of the propagator $L_{p n}$, which includes transitions between the levels near Fermi-surface where smearing effects due to pairing correlations are most essential, is calculated directly according to Eq. (27) and then tranformed to the coordinate representation. Finally, the $\tilde{A}_{p n}$ is the part of the propagator $A$ in the $\lambda$-representation, corrected for the pairing contribution, which must be substracted from the exact propagator $A$ :

$$
\tilde{A}_{p n}(\omega)=\frac{v_{n}^{2}}{-\omega-\delta \mu-E_{n}-\varepsilon_{p}}+\frac{v_{p}^{2}}{\omega+\delta \mu-E_{p}-\varepsilon_{n}} .
$$

Note that the $L, N, K, \tilde{A}$ propagators should be calculated within the same basis which is used for the $\Delta_{\tau}$ and $\mu_{\tau}$ evaluations from Eqs. (20) and (21). Eqs. (20), (29)-(32) solve the problem of the $L\left(\vec{r}, \vec{r}^{\prime} ; \omega\right)$ propagator construction in the $(r, \lambda)$ representation. Actual calculations were carried out with an artificial damping width $\gamma$ introduced in the quasiparticle Green's functions [28] to simulate an experimental resolution and to present the results in a visualized form. Thus all excited states including the bound ones acquire the "damping width" $\Gamma_{d}=4 \gamma$ in addition to the escape width $\Gamma_{\text {esc }}$.

The effective interaction in the particle-hole charge-exchange channel can be written as a sum of two terms: $F^{\omega}=F_{\tau}^{\omega}+F_{\sigma \tau}^{\omega}$. The first term $F_{\tau}^{\omega}$ was obtained as the second functional derivative of the density functional described above with respect to the normal isovector density. The detailes of this derivation can be found in Ref. [29]. The second term, the spin-dependent effective interation $F_{\sigma \tau}^{\omega}$, was chosen as in Ref. [3]. It contains local $\delta$-part with Landau-Migdal parameter $g^{\prime}$ and renormalized one-pion exchange amplitude. In the momentum representation it reads

$$
F_{\sigma \tau}^{\omega}=2 C_{0}\left[g^{\prime} \vec{\sigma}_{1} \vec{\sigma}_{2}-g^{\pi}\left(1-2 \zeta_{s}^{\pi}\right)^{2} \frac{\left(\vec{\sigma}_{1} \vec{k}\right)\left(\vec{\sigma}_{2} \vec{k}\right)}{k^{2}+m_{\pi}^{2}+P_{\Delta}\left(k^{2}\right)}\right],
$$


where $g_{\pi}=-4 \pi\left(f_{\pi}^{2} / m_{\pi}^{2}\right) / C_{0}=-1.45$ and $P_{\Delta}\left(k^{2}\right)$ is the pion irreducible polarization operator in nuclear medium with allowance for virtual production of the $\Delta$ isobar. The constant $g^{\prime}=1.1$ was extracted [3] from the positions of the GT and M1 resonances. The parameter $\zeta_{a}^{\pi}$ characterizes the suppression of spin-isospin vertices in nuclei due to the quasiparticle local charge $[23] e_{q}^{\pi}[\sigma \tau]=1-2 \zeta_{s}^{\pi}$. The value of $\zeta_{a}^{\pi}=\zeta_{s}=0.1$ was determined by a fit to the observed GT and M1 strength distributions [3].

The effective interaction in the particle-particle channel entering Eqs. (3) and (26) for the charge-exchange excitations can be written in a similar fashion as $F^{\xi}=$ $F_{\tau}^{\xi}+F_{\sigma \tau}^{\xi}$ where

$$
\begin{aligned}
& F_{\tau}^{\xi}\left(\vec{r}_{1}, \vec{r}_{2}\right)=-C_{0} f_{\xi}^{\prime} \delta\left(\vec{r}_{1}-\vec{r}_{2}\right), \quad\left(\Delta J^{\pi}=0^{+}, 1^{-}, \ldots\right) ; \\
& F_{\sigma \tau}^{\xi}\left(\vec{r}_{1}, \vec{r}_{2}\right)=-C_{0} g_{\xi}^{\prime} \delta\left(\vec{r}_{1}-\vec{r}_{2}\right), \quad\left(\Delta J^{\pi}=0^{-}, 1^{+}, \ldots\right) .
\end{aligned}
$$

The strength constant $f_{\xi}^{\prime}$ was taken to be equal to the pairing constant $f^{\xi}$ for identical nucleons as it appears in (14). The value of $g_{\xi}^{\prime}=0.2$ for the spin-isospin constant in the $p p$-channel, deduced from the $\beta$-decay data in Ref. [25], was used.

Having solved Eq. (26) the strength function determining the response of a superfluid nucleus to the charge-exchange external field $V_{0}$ can be found:

$$
S(\omega)=\left[\int \hat{e}_{q} V_{0}(\vec{r}) \rho_{t r}(\vec{r} ; \omega) \mathrm{d} \vec{r}\right]^{2}
$$

where the transition density of a nuclear excitation with a frequency $\omega_{\mathrm{g}}$ is calculated in the FFS theory as

$$
\begin{aligned}
\rho_{t r}\left(\vec{r} ; \omega_{s}\right)=C \operatorname{Im} \int \mathrm{d} \vec{r} & {\left[L\left(\vec{r}, \overrightarrow{r^{\prime}} ; \omega_{s}\right) V\left(\overrightarrow{r^{\prime}} ; \omega_{s}\right)+M\left(\vec{r}, \overrightarrow{r^{\prime}} ; \omega_{s}\right) V^{h}\left(\overrightarrow{r^{\prime}} ; \omega_{s}\right)\right.} \\
& \left.+N^{1}\left(\vec{r}, \overrightarrow{r^{\prime}} ; \omega_{s}\right) d^{1}\left(\overrightarrow{r^{\prime}} ; \omega_{s}\right)+N^{2}\left(\vec{r}, \overrightarrow{r^{\prime}} ; \omega_{s}\right) d^{2}\left(\overrightarrow{r^{\prime}} ; \omega_{s}\right)\right],
\end{aligned}
$$

the normalization constant $C$ is calculated through the matrix element of the nuclear transition from the ground state to the excited state:

$$
M_{0 \rightarrow s}^{2}=\left[\int \mathrm{d} \vec{r} e_{q} V_{0}(\vec{r}) \rho_{t r}\left(\vec{r}, \omega_{s}\right)\right]^{2}=\int_{\Delta \omega} S(\omega) \mathrm{d} \omega .
$$

Here $\Delta \omega$ is an energy interval in which the contribution of some specific maximum (or resonance) in $S(\omega)$ at $\omega=\omega_{s}$ can be extracted.

\section{Results and discussion}

\subsection{GT-strength functions for ${ }^{71} \mathrm{Ga}$ and ${ }^{115} \mathrm{In}$}

In this section we present and discuss the results obtained within the self-consistent approach outlined in Sect. 2. The GT matrix elements are calculated with local quasiparticle charge $e_{q}[\sigma \tau]=0.8[3,4]$, i.e. with an effective axial-vector weak interaction constant $g_{A}=1$ (in units of $g_{V}$ ). 
As can be seen from table 1, the calculated matrix elements for the transition between the ground states ${ }^{71} \mathrm{Ga} \rightarrow{ }^{71} \mathrm{Ge}$ and that between the ground state of ${ }^{115} \mathrm{In}$ and the first excited state of ${ }^{115} \mathrm{Sn}$ are in good agreement with experiment. It should be mentioned that in the shell model with configuration mixing [11] these matrix elements are reproduced only if one takes into account higher order ground state correlations (up to $4 p 1 h$ ).

The calculated GT strength distributions for the ${ }^{71} \mathrm{Ga} \rightarrow{ }^{71} \mathrm{Ge}$ and ${ }^{115} \mathrm{In} \rightarrow{ }^{115} \mathrm{Sn}$ GT transitions are shown in Fig. 1 . We notice that in the case of ${ }^{71} \mathrm{Ga}$ the contribution to the sum rule from a "pygmy" resonance, located within the excitation energy region 4-6 MeV, to the sum rule increases by $17 \%$ due to pairing correlations as compared with the $\Delta=0$ approximation. This energy region is very important for neutrino measurements by gallium detector since the excitations above the neutron separation energy $B_{n}=7.46 \mathrm{MeV}$ in the daughter nucleus ${ }^{71} \mathrm{Ge}$ lead to the stable nucleus ${ }^{70} \mathrm{Ge}$ which cannot be registered by radiochemical methods. The total GT strength summed up to $B_{n}$ in ${ }^{71} \mathrm{Ge}$ is $B(\mathrm{GT})=2.15$. In Refs. [11, 21], the corresponding values of $B(\mathrm{GT})=3.90$ and 4.70 were obtained while from the ${ }^{71} \mathrm{Ga}(p, n)^{71} \mathrm{Ge}$ experiment [30] $B(\mathrm{GT})_{\exp }=4.5 \cdot(1 \pm 0.5)$. Thus our calculation produces not enough GT strength in this energy region (see however subsections 3.3 and 3.4).

In the GTR region, at higher excitation energies, the calculated strength distribution reveals two bumps. One of them is due to the $\nu 2 g_{9 / 2}^{-1} \rightarrow \pi 2 g_{9 / 2}$ transition which arises through pairing correlations. This allows us to explain approximately the half of the experimental GTR width [30,34]. Within the excitation energy region up to $20 \mathrm{MeV}$ the GT transitions in $\beta^{-}$-channel contribute $\approx 110 \%$ to the sum rule $\Sigma=3 e_{q}^{2}[\sigma \tau](N-Z)$, while $\approx 10 \%$ of the total GT strength is in the $\beta^{+}$-channel. It should be noted that, in our calculations, the contribution to the sum rule from resonances in the continuum is given by $M_{\mathrm{GT}}^{2}=2 \pi\left(\Gamma_{d}+\Gamma_{\mathrm{esc}}\right) e_{q}^{2}[\sigma \tau] S_{\max }$ where $S_{\max }$ is the maximum of the strength function at $\omega=\omega_{s}$ and $\Gamma_{\text {esc }}$ is the escape width due to direct nucleon emission to the continuum (for example, $\Gamma_{\text {esc }}=64 \mathrm{keV}$ for the resonance at $\omega_{s}=12.2 \mathrm{MeV}$ ).

The strength function for ${ }^{115} \mathrm{Sn}$ calculated in the $\Delta \neq 0$ approximation is shown in the lower part of Fig. 1 where the characteristic regions of the ground state transition, pygmy resonance and GTR can be clearly seen. The excitations in the $\beta^{-}$-channel up to $\omega=25 \mathrm{MeV}$ contribute $91.3 \%$ to the sum rule of $\Sigma=32.64$ when the interaction (34) is taken into account. In addition, $9.6 \%$ of the sum rule is located within the $25<\omega<35 \mathrm{MeV}$ interval and $0.9 \%$ is contained in the $\beta^{+}$-channel. The GT strength integrated up to neutron separation energy $B_{n}=7.56 \mathrm{MeV}$ of the daughter nucleus ${ }^{115} \mathrm{Sn}$ is $B(\mathrm{GT})=2.10$. Within the excitation energy region below $E_{x}=15 \mathrm{MeV}$ the total transition strength is $B(\mathrm{GT})=14$.11. Positions of the main bumps of the calculated GT strength distribution agree quite well with those observed in the $0^{\circ}$ spectrum of the ${ }^{115} \operatorname{In}(p, n){ }^{115} \mathrm{Sn}$ reaction at $E_{p}=120 \mathrm{MeV}[30]$.

To demonstrate the role played by pairing correlations, we have also calculated the strength functions in the $\Delta=0, F^{\xi}=0$ approximation. The energies of the main resonances in the $\beta^{-}$-channel have turned to be in a good agreement with those 
extracted from the ${ }^{71} \mathrm{Ga}(p, n)$ reaction at $E_{p}=35 \mathrm{MeV}$ [34] and $E_{p}=120 \mathrm{MeV}$ [30]. However, the probability of the $\nu 2 p_{3 / 2}^{-1} \rightarrow \pi 2 p_{1 / 2}$ transition between the ground states of ${ }^{71} \mathrm{Ga} \rightarrow{ }^{71} \mathrm{Ge}$, calculated in the $\Delta=0$ approximation, exceeds the experimental one by 2.2 times, and for the transition $\nu 1 g_{9 / 2}^{-1} \rightarrow \pi 1 g_{7 / 2}$ in ${ }^{115} \mathrm{In} \rightarrow{ }^{115} \mathrm{Sn}$, by 2.5 times.

3.2 GT strength distribution in ${ }^{19} \mathrm{~F}$

Calculations for light odd nucleus ${ }^{19} \mathrm{~F}$ reveal some peculiarities. Owing to relatively simple shell structure (3 valence nucleons) this nucleus is an interesting object for nuclear structure theories $[35,36]$. Electron scattering [37] and $\gamma$-decay experiments [38] indicate a strong deformation in the ground state and in the low-lying excited states of ${ }^{19} \mathrm{~F}$. The low-energy spectrum of this nucleus can be described by rotational bands built on the $\mathrm{K}^{\pi}=\frac{1}{2}^{+}$ground state and the $\mathrm{K}^{\pi}=\frac{1}{2}^{-}$first excited state. However, additional levels were found which cannot be explained within this simple scheme. In Refs. $[36,37]$ the ${ }^{19} \mathrm{~F}$ spectrum and charge-exchange transitions to ${ }^{19} \mathrm{Ne}$ were calculated in the shell model approach [10] within $s d$-configuration space $\left(1 d_{5 / 2}, 1 d_{3 / 2}\right.$ and $2 s_{1 / 2}$ subshells were included). The presence of some extra states which are not reproduced in this model indicates both the necessity of basis expansion and possible existence of intruder states.

The approach used in the present paper does not describe the inversion of the $1 d_{5 / 2}$ and $2 s_{1 / 2}$ levels in ${ }^{19} \mathrm{~F}$. Experiment shows that a significant portion of GT strength is contained in the ${ }^{19} \mathrm{~F}\left(\frac{1}{2}^{+}\right) \rightarrow{ }^{19} \mathrm{Ne}\left(\frac{1}{2}^{+}\right)$transition between the ground states. The matrix element is known from $\beta$-decay of ${ }^{19} \mathrm{Ne}$ as $B(\mathrm{GT})=1.62 \pm 0.04$. The weight of the GT component in this mixed transition is $f=0.93 \pm 0.01$ [39]. This means that in the proton as well as in neutron subsystem, the $2 s_{1 / 2}$ states are filled before $1 d_{5 / 2}$. In the presence of the neutron pairing one can expect some portion of the total GT strength to be depleted by the $\nu 1 d_{5 / 2} \rightarrow \pi 1 d_{5 / 2}, \pi 1 d_{3 / 2}$ transitions.

To account for the level inversion in ${ }^{19} \mathrm{~F}$ in the present work an additional correction was introduced in the calculated self-consistent potentials to reproduce the observed ordering of the single-particle levels $\nu s_{1 / 2}, \pi s_{1 / 2}$ and $\nu d_{5 / 2}$ and the energy gaps between them: $\left|\varepsilon\left(\nu 1 d_{5 / 2}\right)-\varepsilon\left(\nu 2 s_{1 / 2}\right)\right|=1.47 \mathrm{MeV},\left|\varepsilon\left(\pi 2 s_{1 / 2}\right)-\varepsilon\left(\pi 1 d_{5 / 2}\right)\right|=0.24$ $\mathrm{MeV},\left(Q_{p n}=3.30 \mathrm{MeV}\right.$ and $\left.Q_{n p}=4.82 \mathrm{MeV}\right)$ [39]. The calculated GT strength distribution for ${ }^{19} \mathrm{~F}$ has three main peaks in the $\beta^{-}$-channel which correspond to the $\nu 2 s_{1 / 2} \rightarrow \pi 2 s_{1 / 2}$ and $\nu 1 d_{5 / 2} \rightarrow \pi 1 d_{5 / 2}, 1 d_{3 / 2}$ transitions. Their energies and $B(\mathrm{GT})$ values, calculated with $e_{q}[\sigma \tau]=1$, are $E_{x 1}=0 \mathrm{MeV}, B(\mathrm{GT})_{1}=1.64, E_{x 2}=5.20$ $\mathrm{MeV}, B(\mathrm{GT})_{2}=0.16$ and $E_{x 3}=7.40 \mathrm{MeV}, B(\mathrm{GT})_{3}=1.40$. In the $\beta^{+}$-channel all the strength comes from the transition $\pi 2 s_{1 / 2} \rightarrow \nu 2 s_{1 / 2}: E_{x}=0 \mathrm{MeV}, B(\mathrm{GT})=0.43$. Thus, in the considered energy region, the sum rule depletion by the excitations in both channels is $93 \%$. The total GT strength in the ${ }^{19} \mathrm{~F} \rightarrow{ }^{19} \mathrm{O}$ channel is $\approx 13 \%$ of the total GT strength in the ${ }^{19} \mathrm{~F} \rightarrow{ }^{19} \mathrm{Ne}$ channel. For the latter our calculation with $e_{q}[\sigma \tau]=0.8$ yields $\sum B(\mathrm{GT}) \approx 2.0$, in excellent agreement with the total GT strength of $1.97 \pm 0.06$ deduced from the ${ }^{19} \mathrm{~F}(p, n){ }^{19} \mathrm{Ne}$ experiment [32]. The main contribution comes from the transition between ground states while the integrated strength of the observed seven GT transitions in the region of $E_{x} \geq 5.4 \mathrm{Mev}$ is $0.35 \pm 0.07$. This may 
indicate the strong fragmentation of the GT strength that is not described by the present method.

3.3 Cross sections of reactor antineutrino capture by ${ }^{71} \mathrm{Ga},{ }^{115} \mathrm{In}$ and ${ }^{19} \mathrm{~F}$

In this section the calculated strength distributions are applied to estimate the reactor antineutrino capture cross sections (assuming $\overline{\nu_{e}} \equiv \nu_{e}$ ). The cross section of $\nu_{e}\left(\bar{\nu}_{e}\right)$-capture (of the inverse $\beta$-decay) in the approximation of allowed transitions is given by

$$
\sigma_{\nu}\left(E_{\nu}\right)=\frac{g_{A}^{2}}{\pi \hbar^{4} c^{3}} \int_{0}^{E_{\nu}-Q} p_{e} E_{e} F\left(Z, E_{e}\right) S_{\beta}(\omega) \mathrm{d} \omega
$$

where $p_{e}$ and $E_{e}$ are the electron momentum and energy, respectively, $F\left(Z, E_{e}\right)$ is the Fermi function and $S_{\beta}(\omega)$ is the strength function of the Fermi and Gamow-Teller $\beta$-transitions. The energy of the emitted electron is given by $E_{e}=E_{\nu}-Q-\omega+m_{e} c^{2}$, where $Q$ is the reaction energy threshold and $\omega$ the excitation energy in daughter nuclei.

The capture cross sections $\sigma_{\nu}\left(E_{\nu}\right)$ as functions of incoming neutrino energy $E_{\nu}$, calculated with the GT strength distributions obtained in the $\Delta \neq 0$ approximation (Fig. 1, solid curves), are plotted in Fig. 2. The cross section $\sigma_{n}$ for ${ }^{71} \mathrm{Ga}$ (curve 1) is calculated with allowance for the GT excitations below the neutron separation energy in daughter nucleus only, with $\sigma_{0}$ (dashed-double-dotted line) corresponding to the transition between the ground states ${ }^{71} \mathrm{Ga} \rightarrow{ }^{71} \mathrm{Ge}$. The cross section for ${ }^{115} \mathrm{In}$ (curve 2) includes all the GT excitations up to $20 \mathrm{MeV}$. It is seen that the cross section $\sigma_{n}$ for ${ }^{71} \mathrm{Ga}$ starts to exceed the cross section $\sigma_{0}$ at $E_{\nu} \approx 3 \mathrm{MeV}$ when the pygmy resonance region becomes available for excitation.

Shown also in Fig. 2 for ${ }^{71} \mathrm{Ga}$ are the cross section $\sigma_{n}$ calculated with the strength function from Ref. [11] (dashed-dotted curve) and the cross section taken from Ref. [21] (dashed line). They both are larger than the corresponding cross section of the present work because the GT strength of Refs. $[11,21]$ summed up to the neutron separation energy in ${ }^{71} \mathrm{Ga}$ is larger. This can be explained by an energy-dependent contribution of the complex configurations (up to $5 p 1 h$ ) which has been taken into account in Ref. [11]. In the lower excitation energy region, $E_{x}<B_{n}$, an additional GT strength arises mainly due to ground state correlations, and the capture cross section increases as compared with the $2 p 1 h$-approximation. At excitation energies in the vicinity of the GTR, the effect of fragmentation over complex configurations results in a shift of some strength to lower energies, around $E_{x} \approx B_{n}$, thus also increasing the cross section $\sigma_{\nu}$.

For the calculations of the total absorption cross sections of reactor antineutrinos an experimental $\overline{\nu_{e}}$ spectrum was used. It has been deduced in Ref. [40] from the measurements of the $\bar{\nu}_{e}+p \rightarrow n+e^{+}$reaction with the Rovno Neutrino Spectrometer (RONS) at the Rovno nuclear power station. The reactor fuel composition corresponds to the following average contribution of the main fission isotopes to the total number of fissions: $59.3 \%$ of ${ }^{235} \mathrm{U}, 28.6 \%$ of ${ }^{239} \mathrm{Pu}, 7.5 \%$ of ${ }^{238} \mathrm{U}$ and $4.7 \%$ of ${ }^{241} \mathrm{Pu}$. 
The deduced $\bar{\nu}_{e}$ spectrum can be approximated as

$$
\rho\left(E_{\bar{\nu}}\right)=6.506 \cdot \exp \left[-\frac{E_{\bar{\nu}}}{1.3307}-\left(\frac{E_{\bar{\nu}}}{7.255}\right)^{2}-1.05\left(\frac{E_{\bar{\nu}}}{8}\right)^{10}\right], \mathrm{MeV}^{-1} \text { fission }^{-1},
$$

where the antineutrino energy is within the $1.95<E_{\bar{\nu}}<9.05 \mathrm{MeV}$ interval. The lower limit is close to the reaction threshold while the upper one is set by a very low $\bar{\nu}_{e}$ flux from the reactor to be measurable. Experimental data on the reactor antineutrino spectrum at lower energies are missing. In the present work, for the $E_{\bar{\nu}} \leq 1.95$ $\mathrm{MeV}$ region, the calculated $\bar{\nu}_{e}$ spectra for fissionable isotopes are used. They were obtained by Vogel et al. [41] by summing the individual $\bar{\nu}_{e}$ spectra occuring in the $\beta$-decay of fission products. The superposition of these spectra, according to the isotope composition listed above, is used. The normalization factor of $\mathrm{N}=1.2$ was introduced for matching it with the RONS spectrum [40] at $E_{\bar{\nu}}=1.95 \mathrm{MeV}$ in such a way that the total number of antineutrino per fission was retained equal to $\approx 6$.

The calculated reactor $\bar{\nu}_{e}$-absorption cross sections are shown in Table 2 . The cross sections related to the soft part of the $\bar{\nu}_{e}$ spectrum $\left(E_{\bar{\nu}} \leq 1.95 \mathrm{MeV}\right)$ are also shown. The contribution from the pygmy resonance region is suppressed owing to the rapid decrease of the RONS $\bar{\nu}_{e}$ spectrum in the region above $E_{\bar{\nu}}=4 \mathrm{MeV}$. Reactor antineutrino capture cross sections are sensitive to the appearance of additional levels of complex nature at low energies $\leq 2 \mathrm{MeV}$. In our calculation, the GT strength in this region is mainly determined by a contribution from the transition between the ground states and, consequently, the cross sections $\bar{\sigma}_{\text {tot }}$ and $\bar{\sigma}_{0}$ differ not too much from each other. It should be also noticed that reactor antineutrino experiments are carried out with discrimination on the energy of outgoing $\beta$-particle. The calculations show that the dependence of the average absorption cross section on the upper discrimination threshold is quite strong (see Fig. 3). This should be taken into account when comparing the calculated cross sections with experimental ones.

3.4 Solar neutrino capture rates for ${ }^{71} \mathrm{Ga}$ and ${ }^{115} \mathrm{In}+{ }^{19} \mathrm{~F}$

The most important sources of the solar neutrinos are the pp-cycle reactions (labeled from 1 to 5 in Table 3 ). Neutrinos with continuous energy spectra are produced via reactions 1,3 and 5 involving $\mathrm{H},{ }^{3} \mathrm{He}$ and ${ }^{8} \mathrm{~B}$ while the reactions 2 and 4 on the $\mathrm{H}$ and ${ }^{7} \mathrm{Be}$ nuclei give monoenergetic neutrinos. The estimated neutrino fluxes and their uncertainties depend on the model of astrophysical evolution of the Sun and on the nuclear input data. They might also be influenced by the vacuum and resonance neutrino oscillations [19]. The solar neutrinos fluxes have usually been estimated within the so called Standard Solar Model [15]. Recently, some new SSM calculations have been published [44, 45, 46, 47].

In the light of recent GALLEX and SAGE results which gave for the first time an experimental evidence for solar neutrino from the pp-cycle it is of interest to discuss the uncertaintes of the calculated neutrino capture rates, which stem from a model dependence of the GT strength distributions. As it was mentioned above, the Ga and In-F detectors are sensitive both to the low and high energy parts of neutrino spectrum. The calculated solar neutrino absorption cross sections for seven most 
important neutrino sources and corresponding rates in gallium and indium-fluorine detectors are presented in Table 3 in comparison with the results of Refs. [11, 15].

It can be seen from Table 3 that more than half of the predicted total rate comes from the pp neutrinos. This contribution is determined by the matrix elements of the GT transition between the lowest allowed partners in ${ }^{71} \mathrm{Ga}$ and ${ }^{115} \mathrm{In}$. Thus the uncertainty regarding the contribution of the pp neutrinos might not be discussed as long as one uses the experimental matrix elements [31, 33]. A slight deviation (2\%) of the $R_{p p}$ calculated in the present work from the $R_{p p}$ values of Refs. $[11,15]$ is explained by the difference in the GT matrix elements used. The same argumentation holds for the ${ }^{7} \mathrm{Be}$ neutrino capture rate but in this case one can expect also some contribution from very low-lying GT exitations, which are not described by the models cited in Table 3. There is an indication on such a low-energy structure $\left(E_{x} \leq 1\right) \mathrm{MeV}$ in the experimental spectra [30]. The use of the experimental strength function for the calculations in Ref. [15] results in a slightly higher ${ }^{7} \mathrm{Be}$ rate (see Table 3 ). This means that the uncertainty of the estimated ${ }^{7} \mathrm{Be}$ neutrino capture rate is higher than that for the pp neutrinos.

The ${ }^{8} \mathrm{~B}$ neutrino capture rate is very model dependent because of sensitivity to the GT strength distribution in the region near the maximum of ${ }^{8} \mathrm{~B}$ neutrino spectrum (7-7.5 MeV). This effect is especially important for ${ }^{71} \mathrm{Ga}$ since the neutron separation energy (7.46 Mev) in ${ }^{71} \mathrm{Ge}$ is a "natural" registration threshold for the gallium detector. All the calculations $[11,21,15]$ give an estimate of $10-13 \%$ for the ${ }^{8} \mathrm{~B}$ contribution to the total capture rate. The ${ }^{8} \mathrm{~B}$ neutrino capture rate for ${ }^{71} \mathrm{Ga}$, obtained with the strength function of Fig. 2, is nearly twice as low compared with calculation of Ref. [15] where the strength function extracted from the $(p, n)$ experiment [30] was used. One of the reason for this difference could be the quasiparticle-phonon coupling not included in our scheme. The expected effect is a redistribution of the GT strength below and above the GTR. In particular, this mechanism is known to shift a part of the GTR strength to lower energies. One of the most extended microscopic models for calculating the response functions of charge-exchange excitations allowing for such an effect is the so called second RPA (SRPA). This scheme accounts for the $2 p 2 h$ correlations in the ground and excited states [48]. However, it has not been applied yet to superfluid nuclei including the odd ones. The development of a QRPA-type scheme with inclusion of the quasiparticle-phonon coupling is still in an initial stage [49]. At the same time this model seems to be rather complicated in practical calculations. A reasonable estimate of the influence of more complex configurations on the strength distributions can be obtained by using an approximate expression for the corresponding contribution to the self-energy operator [48]: $\Sigma_{p h}=\Delta(E)+i \Gamma^{\downarrow}(E) / 2$, where the imaginary part defines a spreading width of the excitation and the real part gives its energy shift. To parametrise $\Gamma^{\dagger}(E)$, one may use empirical data on the decay of hole states as well as systematics of the optical potential imaginary part which is closely related to the decay width of the particle states.

We performed the calculations assuming a simple parametrisation of the spreading width. For the line shape of the individual excitations a Lorentz function was used 
with a width $\Gamma^{\downarrow}$ depending on the excitation energy. It was assumed also that $\Gamma^{\downarrow}=$ $\Gamma_{\exp }=200 \mathrm{keV}$ for the excitations below the particle threshold, and $\Gamma^{\downarrow}=\alpha E^{2}$. in the GTR region (with $\alpha=0.018 \mathrm{Mev}^{-1}$ ) [48]. The calculated GT strength functions are shown in Fig. 4 in comparison with those extracted from the ${ }^{71} \mathrm{Ga}(p, n)^{71} \mathrm{Ge}$ [30] and ${ }^{115} \operatorname{In}(p, n){ }^{115} \mathrm{Sn}$ [32] reactions at $E_{p}=120 \mathrm{MeV}$. It turns out that such a recipe allows for the better description of the experimental strength function of ${ }^{71} \mathrm{Ga}$ in the GTR region $\left(E_{x} \leq 11 \mathrm{MeV}\right)$. The GT strength in the region near $B_{n}$ has increased by $\Delta B(\mathrm{GT})=0.63$ changing the ${ }^{8} \mathrm{~B}$ neutrino capture rate from 6.50 to $8.55 \mathrm{SNU}$ (Table 3). However, the deficit of the GT-strength at $E_{x} \leq 8 \mathrm{MeV}$ in our calculations for ${ }^{71} \mathrm{Ga}$ has resulted in somewhat lower capture rate for the ${ }^{8} \mathrm{~B}$ neutrinos as compared to the calculations of Refs. $[11,15]$. The GT strength function calculated in Ref. [11] is in turn close to the experimental one [32] at $E_{x} \leq 5 \mathrm{MeV}$ but disagrees with it at $E_{x} \geq B_{n}$. The strength function of ${ }^{115} \mathrm{In}$ (Fig. 4) reproduces quite well the experimental one in the region of $E_{x} \leq 12 \mathrm{MeV}$. As a consequence, the ${ }^{8} \mathrm{~B}$ neutrino capture rate is rather close to that of Ref. [15] in which the experimental strength function [32] was used. It should be noted, however, that an error in the GT strength extraction from $(p, n)$ data may arise through the multipole decomposition procedure which is not very accurate for the GTR region. Thus, some additional uncertainty (up to $20 \%$ ) might be assigned to the estimated ${ }^{8} \mathrm{~B}$ neutrino capture rates in Ref. [15] based on the experimental GT strength functions.

Within the approach described in subsection 3.3 we have also calculated the cross section for the ${ }^{8} \mathrm{~B}$ neutrino capture by ${ }^{19} \mathrm{~F}$. For the transition between the ground states both the Fermi and Gamow-Teller matrix elements were included with a mixing factor taken from experiment [39]. The calculated cross section is $\bar{\sigma}=1.8 \cdot 10^{-42}$ $\mathrm{cm}^{2}$ and the corresponding capture rate is $R_{8}=10.4 \mathrm{SNU}$. The contribution of higher energy transitions, namely $\nu 1 d_{5 / 2} \rightarrow \pi 1 d_{5 / 2}, 1 d_{3 / 2}$, to the cross section varies from $0.22 \cdot 10^{-42} \mathrm{~cm}^{2}$ to $0.4 \cdot 10^{-42} \mathrm{~cm}^{2}$ depending on the single-particle level scheme. The calculated total rate for the ${ }^{8} \mathrm{~B}$ neutrino capture is $12.7 \mathrm{SNU}$.

The antineutrino capture cross section (capture rate) in the channel ${ }^{19} \mathrm{~F} \rightarrow{ }^{19} \mathrm{O}$ obtained in the $\bar{\nu}_{e} \equiv \nu_{e}$ approximation is $0.08 \cdot 10^{-42} \mathrm{~cm}^{2}(0.5 \mathrm{SNU})$. In fact, for light odd nuclei such as ${ }^{19} \mathrm{~F}$ our estimates may be oversimplified. In our spherical self-consistent calculations with volume pairing only the ${ }^{19} \mathrm{~F}$ nucleus turns out to be semi-magic. Note that for reproducing the ${ }^{19} \mathrm{~F}$ ground state spin in Ref. [50] this nucleus was treated as a deformed one due to the odd proton influence.

Concluding this section let us briefly discuss the uncertainties of the solar neutrino fluxes obtained within the SSM. First, note that all the most recent SSM codes $[44,45,46,47]$, for the same input parameters, give the same results to within 0.5 SNU $(0.5 \%)$ for the Ga detector [44]. Incorporating new physical effects such as helium diffusion leads to the increase of the total capture rates for gallium by about 3.5 SNU (3\%) and increases the ${ }^{8} \mathrm{~B}$ neutrino flux by $12 \%$ (Ref. [44]). The inclusion of the diffusion of heavy elements is expected to give a further increase of this flux. The solar neutrino fluxes predicted by the SSM are also influenced by the nuclear cross section factors used. For the basic pp-fusion reactions, the corresponding fluxes are 
estimated within the SSM with an accuracy of $2 \%$. On the contrary, the fluxes of the ${ }^{7} \mathrm{Be}$ and ${ }^{8} \mathrm{~B}$ neutrinos are very sensitive to the ${ }^{7} \mathrm{Be}(p, \gamma){ }^{8} \mathrm{~B}$ reaction rate. The cross section of this reaction has been measured at different energies, but the extrapolation to the energy range necessary for the Solar model gives a sizeable error (15\%, see Ref. [15]). The overall uncertainty of the total capture rate predicted by the SSM for gallium experiment has been estimated as $10 \%$ in Ref. [44] and $15 \%$ in Ref. [51].

\section{Concluding remarks}

The Gamow-Teller strength functions of the ${ }^{71} \mathrm{Ga},{ }^{115} \mathrm{In}$ and ${ }^{19} \mathrm{~F}$ as well as the capture rates of solar neutrinos and reactor antineutrinos have been calculated by a self-consistent treatment of the charge-exchange excitations of the superfluid oddA nuclei. This approach is based on the density functional with fractional-linear functions approximating the density dependence of local and finite-range effective forces in the particle-hole channel. Pairing was treated self-consistently using a simple parametrization of the particle-particle force; the blocking effect was taken into account. The dynamical QRPA-like equations of the Migdal theory of finite Fermi systems were solved using a spin-dependent charge-exchange interaction containing the local Landau-Migdal force $g^{\prime}$ and a renormalized one-pion exchange amplitude; the particle-hole continuum was included completely. The effect of complex configurations beyond the QRPA was simulated by using an energy-dependent spreading width. The results for the solar neutrino capture rates on ${ }^{71} \mathrm{Ga}$ and ${ }^{115} \mathrm{In}$ compare well with other calculations though are slightly lower (see Table 3). We have also made a simple estimate of the solar neutrino and reactor antineutrino capture rates for the ${ }^{19} \mathrm{~F}$ nuclei.

The observed deviation of the total solar neutrino capture rates from theoretical estimations can not be removed by re-examining the nuclear stucture input because the main contribution coming from the pp and, to a considerable extent, from the ${ }^{7} \mathrm{Be}$ neutrinos are defined by the GT transitions between the lowest allowed states. The corresponding matrix elements are known experimentally and this contribution is evaluated quite accurately by all existing calculations. The contribution from the ${ }^{8} \mathrm{~B}$ neutrinos with excitation of higher lying states is very sensitive to the fragmentation models of the GT strength near the neutron separation threshold in daughter nuclei. However, this contribution to the total capture rate does not exceed $10-13 \%$ so that the corresponding uncertainty in the total rate is expected to be relatively low (3$5 \%)$. The $(p, n)$ experiments with polarized nucleons on ${ }^{71} \mathrm{Ga},{ }^{115} \mathrm{In}$ and ${ }^{19} \mathrm{~F}$ would be useful since the combined microscopic analysis [52] of inclusive neutron spectra and polarization tranfer data could provide a more reliable reconstruction of the GT strength functions at higher excitation energies.

The program of solar neutrino spectroscopy using an In-F detector is very attractive because it suggests the reliable measurements both of the pp and ${ }^{7} \mathrm{Be}$ and of the ${ }^{8} \mathrm{~B}$ neutrino events. This is very actual in the light of the recent GALLEX and 
SAGE data. It also gives the possibility for searching the hypothetical solar antineutrinos and of studing the anomalous channel contribution in reactor experiments. The recent solar neutrino measurements require a critical analysis of the models of formation of both low- and high-energy parts of the neutrino flux. Decisive at this point would be also other planned experiments: detection of the pp neutrinos (neutrino scattering in liquid helium [53]), detection of the ${ }^{7} \mathrm{Be}$ neutrinos (neutrino scattering in a purified liquid scintillator [54], BOREXINO) and detection of the ${ }^{8} \mathrm{~B}$ neutrino (neutrino-induced deutron disintegration in $\mathrm{D}_{2} \mathrm{O}$ [55], SNO). The latter is especially important because it would allow to deduce the central temperature of the Sun to a few percent.

Concerning nuclear stucture theory, an extention of the QRPA-type approaches (incorporating, for example, the quasiparticle-phonon coupling) with application to the collective states of odd nuclei in the charge-exchange channel seems to deserve further development. The practical yield of such an extention would be a more detailed description of the neutrino capture processes. In particular, it could provide a reasonable estimate of the $\gamma$ transitions from excited states to the $\frac{7}{2}^{+}$state in ${ }^{115} \mathrm{Sn}$, which is important for the analysis of signals from an In-F detector. The ordering of the single-particle levels in light odd nuclei $\left({ }^{19} \mathrm{~F}\right.$, etc.) is another interesting problem. It seems that much attention should be paid to the density-dependent pairing correlations in such nuclei. This problem will be considered elsewhere.

\section{Acknowledgements}

The authors thank Professor J.-F. Cavaignac, Drs. A.I. Vdovin and M.D. Skorokhvatov for useful discussions and express their gratitude to Professor L.A. Mikaelyan for encouragement and valuable comments. Two of us (S.F. and I.B.) would like to thank kind hospitality of theory group at GANIL, Caen, as well as of the Max-Plank Institut für Astrophysik, Garching, where this work was completed. We are also grateful to Drs. P. van Isacker and K. Takahashi for critical reading the manuscript and much useful advice.

This work was supported in part by the Russian Fundamental Science Foundation and by the International Science Foundation.

\section{References}

[1] D.H. Wilkinson, Nucl.Phys. A209 (1973) 470.

[2] C. Gaarde, in Nuclear structure, eds. R. Broglia, G.B. Hagemann and B.Herskind (North Holland, Amsterdam, 1985), p. 449.

[3] N.I. Pyatov and S.A. Fayans, Fiz. Elem. Chastits At. Yadra 14 (1983) 953 [Sov. J. Part. Nucl. 14 (1983) 401].

[4] F.A. Gareev, S.N. Ershov, N.I. Pyatov and S.A. Fayans, Fiz. Elem. Chastits At. Yadra 19 (1988) 864 [Sov. J. Part. Nucl. 19 (1988) 373]. 
[5] B.A. Brown and B.H. Wildenthal, At. Data Nucl. Data Tables 33 (1985) 347.

[6] O. Klepper and K. Rykaczewski, Proc. Predeal Int. Summer School. on Recent Adv. in Nucl. Structure (Predeal, Romania, 1990),p. 135.

[7] T. Sekine, I. Cerny, R. Kirchner, O. Klepper, V.T. Koslowsky, A. Plochocki, E. Roeckl, D. Schardt, B. Sherrill and B.A. Brown, Nucl. Phys. A467 (1987) 93; J. Szerypo, D. Bazin, B. A. Brown, D. Guillemaud-Mueller, H. Keller, R. Kirchner, O. Klepper, D. Morrissey, E. Roeckl, D. Schardt and B. Sherrill, Nucl. Phys. A528 (1991) 203.

[8] E.G. Adelberger, A. Garcia, P.V. Magnus and D.P. Wells, Phys. Rev. Lett. 67 (1991) 3658.

[9] H. Miyatake, K. Ogawa, T. Shinouka and M. Fujioka, Nucl. Phys. A470 (1987) 328.

[10] B.H. Wildenthal, Prog. Part. Nucl. Phys. 11 (1984) 5.

[11] C.J. Mathews, S.D. Bloom, G.M. Fuller and J.N. Bahcall, Phys. Rev. C32 (1985) 796.

[12] I.N. Borzov and E.L. Trykov, Yad. Fiz. 52 (1990) 52 [Sov. J. Nucl. Phys 52 (1990) 33].

[13] B. Alex Brown, Phys. Rev. Lett. 69 (1992) 1034.

[14] J. Rapaport and E.R. Sugarbaker, Phys. Rev. Lett. 69 (1992) 2444; C.D. Goodman, M.B. Aufderheide, S.D. Bloom and D.A. Resler, ibid, 2445.

[15] J.N. Bahcall, Phys. Rev. B135 (1964) 137; Rev. Mod. Phys. 54 (1982) 767; J.N. Bahcall and R.K. Ulrich, Rev. Mod. Phys. 60 (1988) 297.

[16] V.A. Kuzmin and G.T. Zatsepin, In Neutrino-88, v. 2 (Moscow, 1988), p. 156.

[17] T. Kirsten et al., 86 Massive Neutrinos in Astrophysics and Particle Physics, eds. O. Facker and Tran Than Van (Paris, 1986), p. 119.

[18] M. Avenier et al., Proc. Int. Conf. on Underground Physics (Toledo, Spain, Sept. 1991).

[19] S.P. Mikheev and A.Yu. Smirnov, Yad. Fiz. 42 (1985)1441 [Sov. J. Nucl. Phys. 42 (1985) 913].

[20] S.D. Bloom and G.M. Fuller, Nucl. Phys. A440 (1984) 511.

[21] K. Grotz, H.V. Klapdor and I. Metzinger, Phys. Rev. 33 (1986) 1263. 
[22] A.A. Borovoy, Yu.S. Lutostansky, S.Kh. Khakimov and N.S. Shulgina, Pis'ma ZhETF 45 (1987) 521 [JETP Lett. 45 (1987) 409].

[23] A.B. Migdal, Theory of Finite Fermi Systems and Applications to atomic nuclei, transl. of 1st Russ. ed. (Interscience, New-York, 1967) [Russ. original, 2nd ed., Nauka, Moscow, 1983].

[24] V.G. Guba, M.A. Nikolaev and M.G. Urin, Proc. 14th LINP Winter School (Liningrad, 1989), p. 364.

[25] I.N. Borzov, E.L. Trykov and S.A. Fayans, Yad. Fiz. 52 (1990) 985 [Sov. J. Nucl. Phys. 52 (1990) 627].

[26] A.P. Platonov and E.E. Saperstein, Nucl. Phys. A486 (1988) 118.

[27] A.V. Smirnov, S.V. Tolokonnikov and S.A. Fayans, Yad. Fiz. 48 (1988) 1661 [Sov. J. Nucl. Phys. 48 (1988) 995].

[28] I.N. Borzov and S.A. Fayans, Preprint FEI-1129, Obninsk, 1981.

[29] S.A. Fayans, E.L. Trykov and D. Zawischa, Nucl. Phys. A, in press.

[30] D. Krofcheck, E. Sugarbaker, I. Rapaport, D. Wang, J.N. Bahcall, R.C. Byrd, C.C. Foster, C.D. Coodman, I.J. Van Heerden, C. Caarde, J.S. Larsen, D.J. Horen, and T.N. Taddeucci, Phys. Rev. Lett. 55 (1985) 1051.

[31] K.R. Alvar, Nucl. Data Sheets 10 (1973) 205.

[32] I. Rapaport, P. Welch, J. Bahcall, E. Sugarbarker, T.N. Taddeucci, C.D. Coodman, C.F. Foster, D. Horen, C. Caarde, J. Larsen, and T. Masterson, Phys. Rev. Let. 54 (1985) 2325.

[33] R.S. Raghavan, Phys. Rev. Lett. 37 (1976) 259.

[34] A.I. Baltz, I. Weneser, B.A. Brown and J. Rapaport, Phys. Rev. Lett. 53 (1984) 2078.

[35] J.P. Elliott and B.H. Flowers, Proc. R. Soc. London, 536 (1955) 64; M.G. Redlich, Phys. Rev. 99 (1955) 1426.

[36] E.G. Adelberger, M.M. Hindi, C.D. Hoyle, H.E. Swanson, R.D. Von Lintig and W.C. Haxton, Phys. Rev. 27 (1983) 2833.

[37] B.A. Brown, B.H. Wildenthal, C.F. Williamson, F.N. Rad, S. Kowalski, Hall Grannel and J.T. O'Brien, Phys. Rev. C32 (1985) 1127.

[38] P.H. Stelson and F.K. McGowen, Nucl. Phys. 16 (1960) 92. 
[39] F. Ajzenberg-Selove, Nucl.Phys. A475 (1985) 1.

[40] Yu.V. Klimov, V.I. Kopeikin, A.A. Labzov, L.A. Mikaelyan, K.V. Ozerov, V.V. Sinev and S.V. Tolokonnikov, Yad. Fiz. 52 (1990) 1574 [Sov. J. Nucl. Phys. 52 (1990) 994].

[41] P. Vogel and J. Engel, Phys. Rev. D39 (1989) 3378.

[42] GALLEX Collaboration, P. Anselmann et al., Phys. Lett. B285 (1992) 376.

[43] SAGE Collaboration, A.I. Abazov et al., Phys. Rev. Let. 67 (1991) 3332.

[44] J.N. Bachcall and M.H. Pinsonneault, Rev. Mod. Phys. 64 (1992) 885.

[45] S. Turck-Chièze, contribution to the Moriond Workshop on Massive Neutrinos and Test of Fundamental Symmetries, Jan. 1992, submitted to Phys. Reports.

[46] P. Morel at al., IAU Colloquium 197 "Inside the Sun", Vienna 1992, submitted to Astron. and Astrophys.

[47] D.B. Guenter et al., Astrophys. J. 387 (1991) 377.

[48] S. Drozdz, S. Nishizaki, J. Speth and J. Wambach, Phys. Repts. 197, (1990) 1.

[49] Liang Zhao, A. Sustich and B.A. Brown, Ann. Phys. 213 (1992) 378.

[50] L. Zetta and L. Zuffi, Nuov. Cim. A2 (1978) 141.

[51] D.R.O. Morrison, Int. J. Mod. Phys. 2 (1992) 281.

[52] I.N. Borzov, F.A. Gareev, S.N. Ershov, R.S. Kurmanov, E.L. Trykov and S.A. Fayans, Yad. Fiz. 55 (1992) 107 [Sov. J. Nucl. Phys. 55 (1992) 60].

[53] R.E. Lanou, H.E. Maris and G.M. Seidel, Phys. Rev. Lett. 58 (1987) 2498.

[54] R.S.Ragahavan, Proc. 25th Int. Conf. High Energy Physics, eds. K.K.Phua and Y. Yamaguchi (World Scientific, Singapore), vol. I, p. 482.

[55] SUDBURY Neutrino Observatory Collaboration, G. Aardsma et al., Phys. Lett. 194 (1987) 321. 


\section{$5 \quad$ Figure captions}

Fig. 1. GT-strength functions for ${ }^{71} \mathrm{Ga} \rightarrow{ }^{71} \mathrm{Ge}$ (upper part) and ${ }^{115} \mathrm{In} \rightarrow{ }^{115} \mathrm{Sn}$ (lower part) calculated in the $\Delta \neq 0$ approximation with interactions of eqs. (33) and (34) (solid lines) and without them (dashed lines).

Fig. 2. Neutrino capture cross sections for ${ }^{71} \mathrm{Ga}$ and ${ }^{115} \mathrm{In}$ as functions of $E_{\nu}{ }^{71} \mathrm{Ga}$ : for the g.s. $\rightarrow$ g.s (dashed-double-dotted line); including all GT-transitions up to the neutron separation threshold $B_{n}$ in ${ }^{71} \mathrm{Ge}$ (curve 1); calculated using the strength function from Ref. [11] (dashed-dotted line); taken from Ref. [21] (curve 2). ${ }^{115}$ In: including all GT-transitions up to $E_{x}=20 \mathrm{MeV}$ (dashed line).

Fig. 3. Total reactor antineutrino absorption cross section on ${ }^{115}$ In as a function of the $\beta$-particle detection threshold.

Fig. 4. GT-strength functions for ${ }^{71} \mathrm{Ga}$ (upper part) and ${ }^{115} \mathrm{In}$ (lower part) extracted from the $(\mathrm{p}, \mathrm{n})$ reactions at $E_{p}=120 \mathrm{MeV}[30,32]$ in comparison with the calculated ones in the $\Delta \neq 0$ approximation (see text). 
Table 1 Calculated and experimental Gamow-Teller matrix elements for the ${ }^{71} \mathrm{Ga} \rightarrow{ }^{71} \mathrm{Ge}\left(\frac{3}{2}^{-} \rightarrow \frac{1}{2}^{-}\right)$and ${ }^{115} \mathrm{In} \rightarrow{ }^{115} \mathrm{Sn}\left(\frac{9}{2}^{+} \rightarrow \frac{7}{2}^{+}\right)$transitions

\begin{tabular}{c|cccccc}
\hline $\begin{array}{c}\text { Nuclei } \\
\text { (Transition) }\end{array}$ & \multicolumn{5}{c|}{$M_{\mathrm{GT}}^{2}$, calc } & \multicolumn{2}{c}{$M_{\mathrm{GT}}^{2}, \exp$} \\
\hline & $\Delta=0$ & $\Delta \neq 0$ & Ref.[11] & Ref.[21] & (pn) & $\beta^{ \pm}$ \\
Ga $\rightarrow \mathrm{Ge}$ & 0.20 & 0.08 & 0.052 & 0.071 & 0.083 Ref.[30] & 0.09 Ref.[31] \\
$\left(\nu 2 p_{3 / 2}^{-1} \rightarrow \pi 2 p_{1 / 2}\right)$ & & & & & & \\
In $\rightarrow \mathrm{Sn}$ & 0.35 & 0.15 & - & - & 0.17 Ref.[32] & 0.154 Ref.[33] \\
$\left(\nu 1 g_{9 / 2}^{-1} \rightarrow \pi 1 g_{7 / 2}\right)$ & & & & & & \\
\hline
\end{tabular}

Table 2 Cross sections for the reactor $\bar{\nu}_{e}$ capture by ${ }^{71} \mathrm{Ga}$ and ${ }^{115} \mathrm{In}+{ }^{19} \mathrm{~F}$

\begin{tabular}{lccc}
\hline $\begin{array}{l}\text { Daughter } \\
\text { nucleus }\end{array}$ & $\begin{array}{c}\text { Energy interval } \\
E_{\bar{\nu}}, \mathrm{MeV}\end{array}$ & $\begin{array}{c}\bar{\sigma}_{0}, \\
10^{-43} \mathrm{~cm}^{2} / \text { fiss. }\end{array}$ & $\begin{array}{c}\bar{\sigma}_{\text {tot }}, \\
10^{-43} \mathrm{~cm}^{2} / \text { fiss. }\end{array}$ \\
\hline${ }^{71} \mathrm{Ge}$ & $0.236-1.95$ & 0.37 & $0.38(0.43)$ \\
& $1.95-9.05$ & 0.89 & $2.00(2.66)$ \\
& & $\Sigma=1.17$ & $\Sigma=2.38(3.09)$ \\
${ }^{115} \mathrm{Sn}$ & $0.119-1.95$ & 1.61 & 1.65 \\
& $1.95-9.05$ & 3.25 & 3.47 \\
& & $\Sigma=4.86$ & $\Sigma=5.12$ \\
${ }^{19} \mathrm{Ne}$ & $3.30-9.05$ & 0.72 & 0.73 \\
${ }^{19} \mathrm{O}$ & $6.28-9.05$ & 0.47 & 0.003 \\
\hline
\end{tabular}

The full $\bar{\nu}_{e} \rightarrow \nu_{e}$ conversion on the target is assumed. Bracketed values are the cross sections calculated with the GT strength distribution taken from Ref. [11]. The cross sections $\overline{\sigma_{0}}$ in the third column correspond to the g.s. $\rightarrow$ g.s. transitions for ${ }^{71} \mathrm{Ga}$ and ${ }^{19} \mathrm{~F}$ and to the $\nu 1 g_{9 / 2} \rightarrow \pi 1 g_{7 / 2}$ transition for ${ }^{115} \mathrm{In}$. 
Table 3 Solar neutrino fluxes and capture rates (in SNU) for the Gallium and Indium detectors

\begin{tabular}{|c|c|c|c|c|c|c|c|}
\hline \multirow{2}{*}{$\begin{array}{l}\text { Neutrino } \\
\text { source }\end{array}$} & \multirow{2}{*}{$\begin{array}{l}E_{\nu}^{\max } \\
\left(\Phi_{i}\right)\end{array}$} & \multicolumn{4}{|c|}{${ }^{71} \mathrm{Ga}$} & \multicolumn{2}{|c|}{${ }^{115} \mathrm{In}$} \\
\hline & & this work & Ref.[11] & Ref.[15] & Ref.[21] & this work & Ref.[15] \\
\hline 1. $\mathrm{H}\left(p, e^{+} \nu_{e}\right)^{2} \mathrm{H}$ & $\begin{array}{l}0.420 \\
(6.00)\end{array}$ & 71.5 & 70.2 & 70.8 & 71.3 & 460 & 468 \\
\hline 2. $\mathrm{H}\left(p e^{-}, \nu_{e}\right)^{2} \mathrm{H}$ & $\begin{array}{l}1.442(d) \\
(0.014)\end{array}$ & 1.8 & 3.0 & 3.0 & 2.5 & 7.7 & 8.1 \\
\hline 3. ${ }^{3} \mathrm{He}\left(p, e^{+} \nu_{e}\right)^{4} \mathrm{Li}$ & $\begin{array}{l}18.795 \\
\left(7.6 \cdot 10^{-7}\right)\end{array}$ & 0.03 & - & 0.06 & - & 0.05 & 0.05 \\
\hline 4. ${ }^{7} \mathrm{Be}\left(e^{-}, \nu_{e}\right){ }^{7} \mathrm{Li}$ & $\begin{array}{l}0.862(d) \\
0.384(d) \\
(0.47)\end{array}$ & 31.1 & 31.2 & 34.3 & 31.2 & 119.8 & 116 \\
\hline 5. ${ }^{8} \mathrm{~B}\left(e^{+}, \nu_{e}\right){ }^{8} \mathrm{Be}$ & $\begin{array}{l}15.00 \\
\left(5.7 \cdot 10^{-4}\right)\end{array}$ & 8.5 & 11.6 & 14.0 & 17.2 & 14.9 & 14.4 \\
\hline 6. ${ }^{13} \mathrm{~N}\left(e^{+}, \nu_{e}\right){ }^{13} \mathrm{C}$ & $\begin{array}{l}1.199 \\
(0.061)\end{array}$ & 2.4 & 3.3 & 3.8 & 2.9 & 13.4 & 13.6 \\
\hline 7. ${ }^{15} \mathrm{O}\left(e^{+}, \nu_{e}\right)^{15} \mathrm{~N}$ & $\begin{array}{l}1.732 \\
(0.052)\end{array}$ & 3.8 & 4.6 & 6.1 & 4.0 & 18.0 & 18.5 \\
\hline Total & $\Sigma R_{i}=$ & 119 & 124 & 132 & 129 & 634 & 639 \\
\hline Experiment & $\begin{array}{l}\text { GALLEX } \\
\text { SAGE }\end{array}$ & $\begin{array}{c}83 \\
\leq 79\end{array}$ & \pm 19.5 [st] & \pm 8 [sys] & $\begin{array}{l}\text { Ref.[42] } \\
\text { Ref.[43] }\end{array}$ & & \\
\hline
\end{tabular}

The maximum neutrino energies $E_{\nu}^{\max }$ (in $\mathrm{MeV}$ ) and the neutrino fluxes $\Phi_{i}$ at the Earth (in $10^{10} \mathrm{~cm}^{-2} \mathrm{~s}^{-1}$ ) for the main solar neutrino sources, as predicted within the SSM by Bahcall and Ulrich [15], are given. 1 SNU $=1$ neutrino capture reaction per second in $10^{36}$ target atoms. 

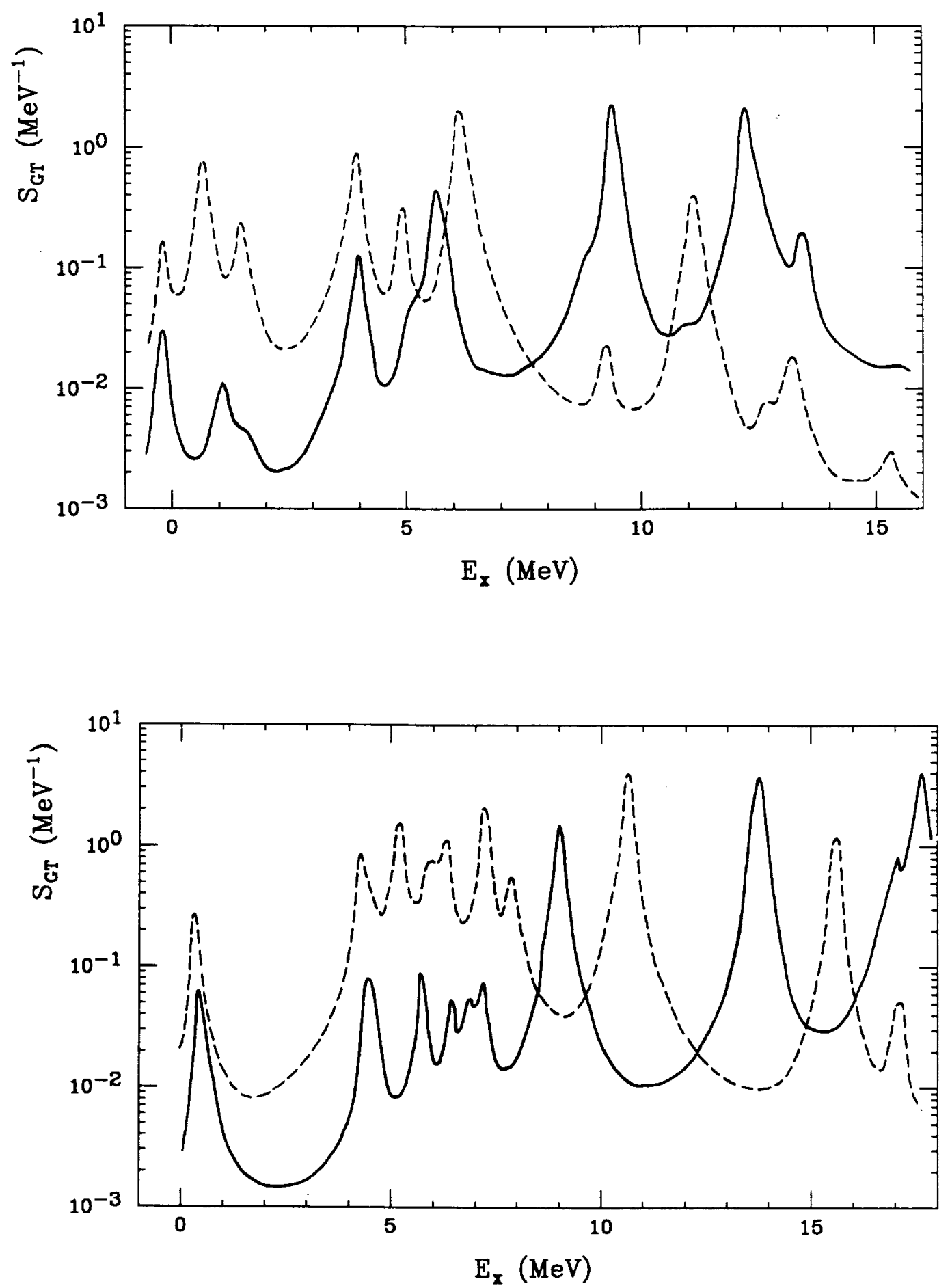

Fig. 1 


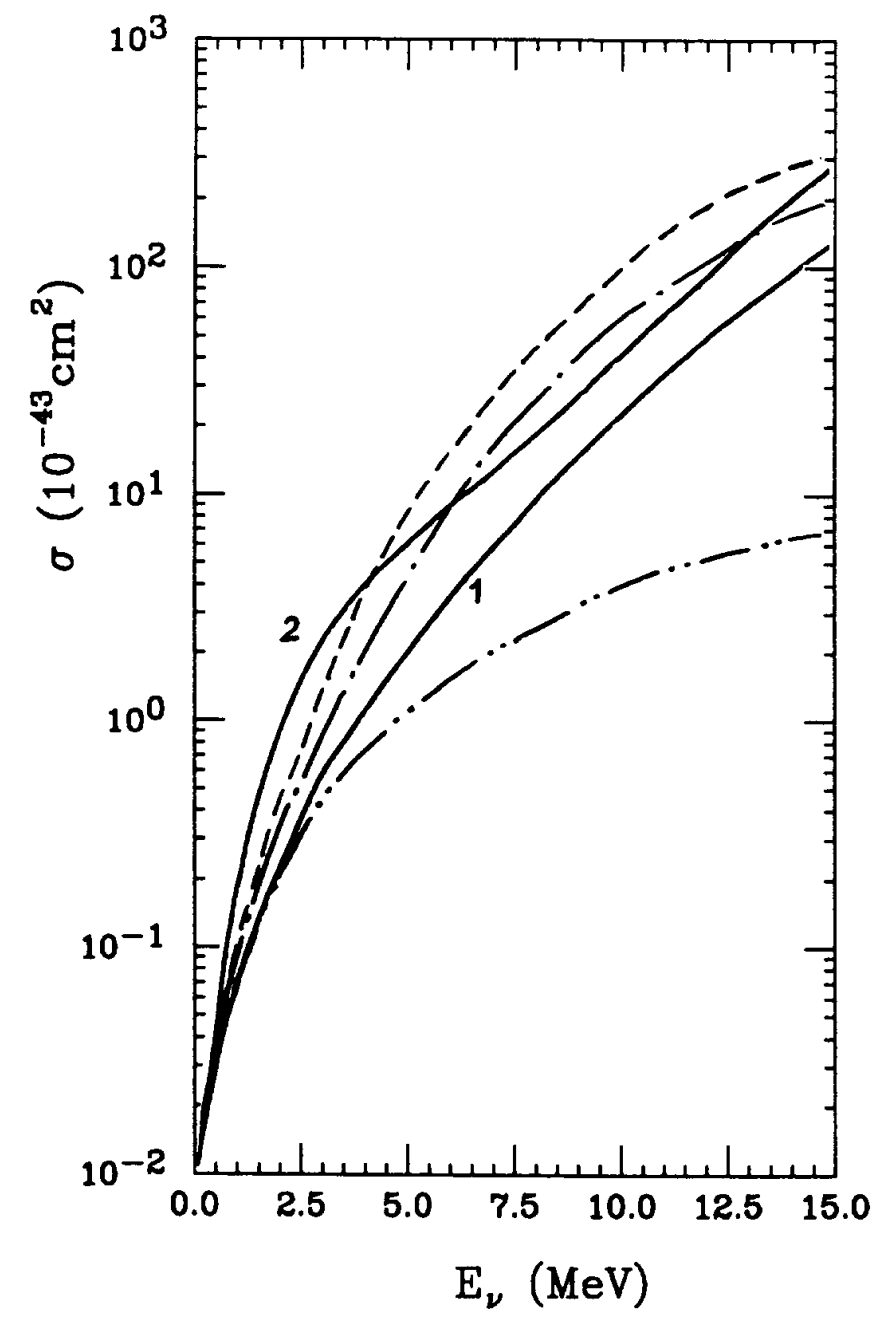

Fig. 2 


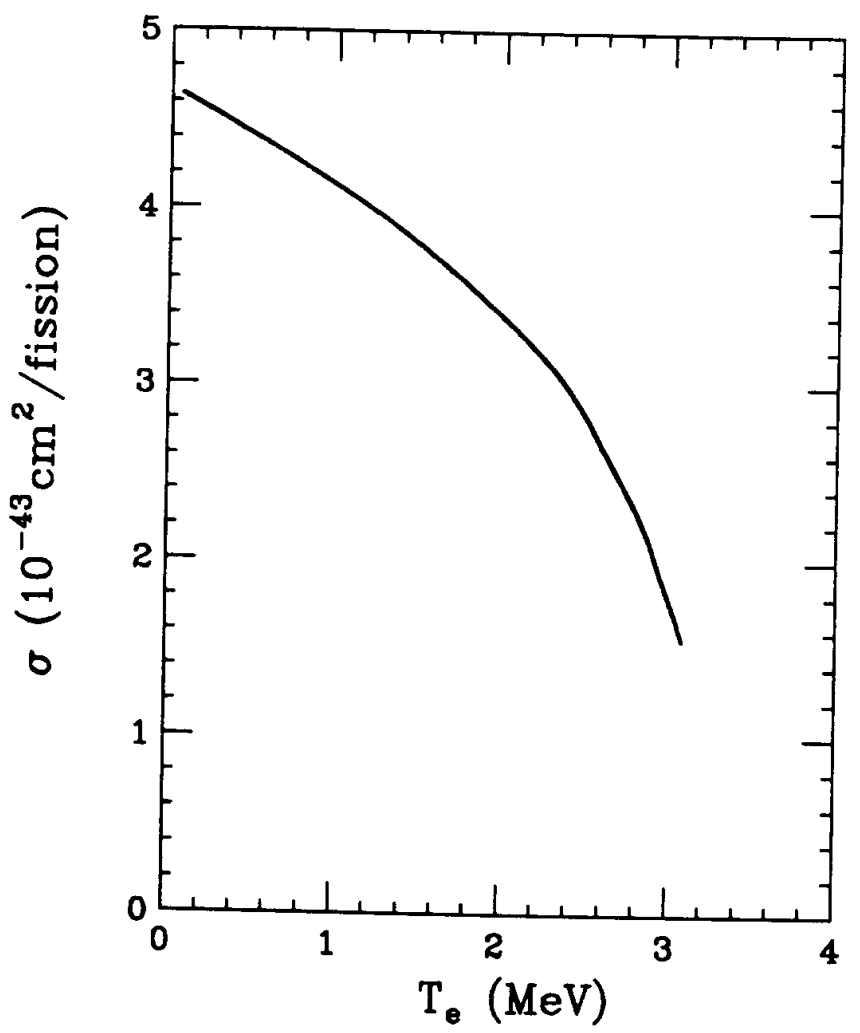

Fig. 3 

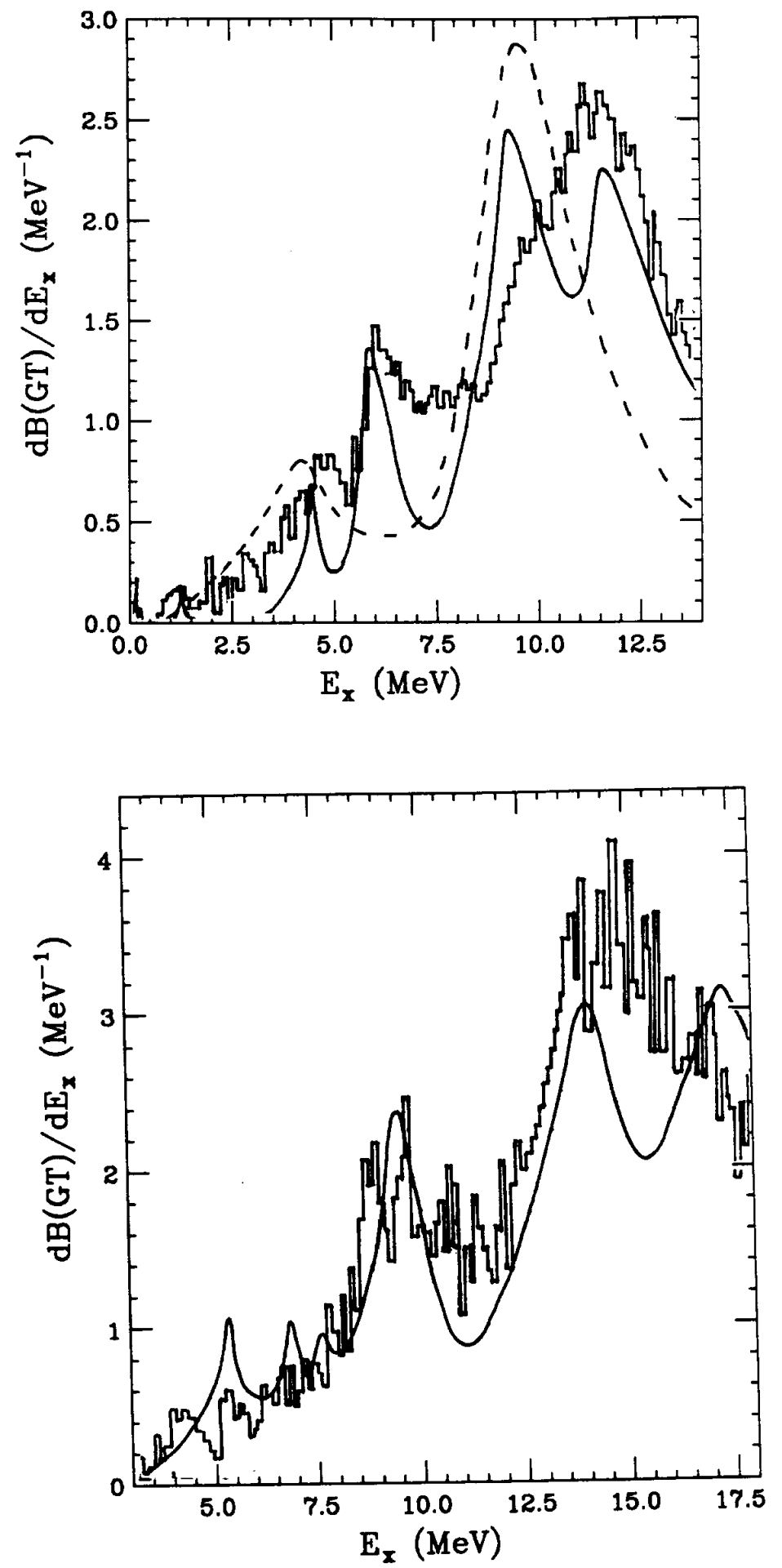

Fig. 4 